\title{
Geochemistry and petrogenesis of Proterozoic granitic rocks from northern margin of the Chotanagpur Gneissic Complex (CGC)
}

\author{
Bhupendra S Yadav ${ }^{1, *}$, Nishchal Wanjari ${ }^{2}$, Talat Ahmad ${ }^{1,3}$ and Rajesh Chaturvedi ${ }^{4}$ \\ ${ }^{1}$ Department of Geology, University of Delhi, Delhi 110 007, India. \\ ${ }^{2}$ Department of Geology, Sikkim University, Gangtok 737 102, India. \\ ${ }^{3}$ Jamia Millia Islamia University, New Delhi, Delhi 110 025, India. \\ ${ }^{4}$ Geological Survey of India, Lucknow 226 024, India. \\ ${ }^{*}$ Corresponding author. e-mail: yadav.bhupendra07@gmail.com
}

This study presents the geochemical characteristics of granitic rocks located on the northern margin of Chotanagpur Gneissic Complex (CGC), exposed in parts of Gaya district, Bihar and discusses the possible petrogenetic process and source characteristics. These granites are associated with Barabar Anorthosite Complex and Neo-proterozoic Munger-Rajgir group of rocks. The granitic litho-units identified in the field are grey, pink and porphyritic granites. On the basis of geochemical and petrographic characteristics, the grey and pink granites were grouped together as GPG while the porphyritic granites were named as PG. Both GPG and PG are enriched in $\mathrm{SiO}_{2}, \mathrm{~K}_{2} \mathrm{O}, \mathrm{Na}_{2} \mathrm{O}, \mathrm{REE}$ (except Eu), $\mathrm{Rb}$, $\mathrm{Ba}, \operatorname{HFSE}\left(\mathrm{Nb}, \mathrm{Y}, \mathrm{Zr}\right.$ ), depleted in $\mathrm{MgO}, \mathrm{CaO}, \mathrm{Sr}$ and are characterised by high $\mathrm{Fe}^{*}$ values, $\mathrm{Ga} / \mathrm{Al}$ ratios and high $\mathrm{Zr}$ saturation temperatures $\left(\mathrm{GPG}_{\mathrm{avg}} \sim 861^{\circ} \mathrm{C}\right.$ and $\left.\mathrm{PG}_{\mathrm{avg}} \sim 835^{\circ} \mathrm{C}\right)$. The REE patterns for GPG are moderately fractionated with an average $(\mathrm{La} / \mathrm{Yb})_{\mathrm{N}} \sim 4.55$ and $\mathrm{Eu} / \mathrm{Eu}^{*} \sim 0.58$, than $\mathrm{PG}$ which are strongly fractionated with an average $(\mathrm{La} / \mathrm{Yb})_{\mathrm{N}} \sim 31.86$ and $\mathrm{Eu} / \mathrm{Eu}^{*} \sim 0.75$. These features indicate that the granites have an A-type character. On the basis of geochemical data, we conclude that the granites are probably derived from a predominant crustal source with variable mantle involvement in a post-collisional setting.

\section{Introduction}

The Proterozoic Eon is marked by the voluminous addition of granitic plutons along the continental arcs and the study of their genesis holds the key to our understanding of the evolution of continental crust (Kemp and Hawkesworth 2003; Frost and Frost 2013). The Chotanagpur Gneissic Complex (CGC) in the eastern Precambrian tract of peninsular India has experienced many episodes of felsic magmatism during the Proterozoic. This is evident from a variety of Proterozoic felsic rocks exposed in different parts of CGC in the form of gneisses and granites. As per Mahadevan (2002), widespread granitic activity in CGC occurred during 1100-1300 and 1600-1500 Ma.

The northern margin of CGC comprises of Bihar Mica Belt (BMB), the Munger Group and the Rajgir-Gaya sub-basins, showing a basement-cover relationship with the CGC gneisses, though most of the field relationship is obscured by the Quaternary alluvium (figure 1) (Ghose and Mukherjee 2000). The study area at the northern margin of CGC near Gaya, Bihar (figure 1 and 2) exposes

Keywords. Chotanagpur Gneissic Complex (CGC); A-type granites; post-collisional. 


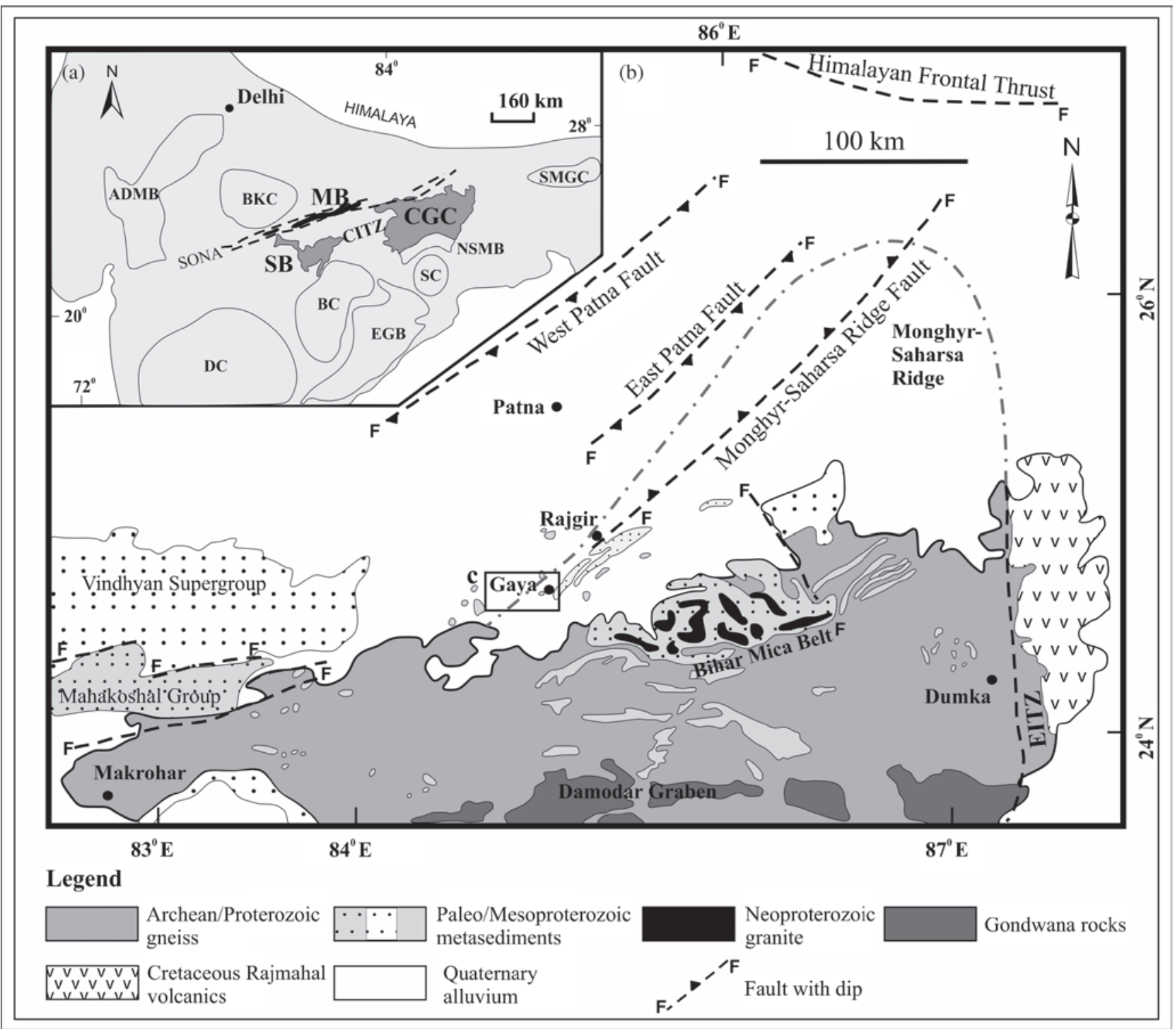

Figure 1. (a) Outline map of India showing the location of different cratons and mobile belts with respect to Chotanagpur Gneissic Complex (CGC). Abbreviations. SB: Satpura Belt; MB: Mahakoshal Belt; ADMB: Aravalli-Delhi Mobile Belt; NSMB: North Singhbhum Mobile Belt; EGB: Eastern Ghats Belt; SMGC: Shillong Meghalaya Gneissic Complex; SONA: Son Narmada Graben; BKC: Bundelkhand Craton; SC: Singhbhum Craton; BC: Bastar Craton; DC: Dharwar Craton. (b) Geological map of the northern part of CGC and adjoining regions showing the location of the study area in the box marked 'c' (after Chatterjee and Ghose 2011). The tectonic features are marked after Sastri et al. (1971), Rao (1973), Valdiya (1976) and GSI (2000).

granitic plutons which are composed of different types of granitic litho-units, related pegmatite and quartz veins (figure 2). Chatterjee and Ghose (2011) reported a crystallisation age of $1697 \pm$ 17 Ma for monazites within the porphyritic granites from Barabar Hills near Gaya which confirms the Proterozoic age of granitic plutons in the region. Wanjari et al. (2012) too have reported an $\mathrm{Rb}-\mathrm{Sr}$ whole rock isochron age of $1337 \pm 26 \mathrm{Ma}$ for the granites from Paharpur near Gaya (figure 2). The available geochronological data from other parts of CGC based on $\mathrm{Rb}-\mathrm{Sr}$ systematics yield similar ages for gneiss from central $(1717 \pm 102 \mathrm{Ma}$,
Mallik et al. 1991) and granites from the western

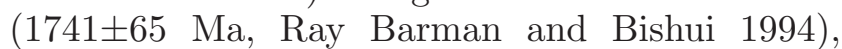
northern (1590 $\pm 30 \mathrm{Ma}$, Pandey et al. 1986) and eastern (1599 \pm 33 Ma, Mallik et al. 1991) parts, which marks the oldest time span of felsic magmatism. However, the last significant granitic and pegmatitic activity marked by $\mathrm{Rb}-\mathrm{Sr}$ dates is between 950 and 800 Ma (Mahadevan 2002). In this paper, we present a new whole rock major and trace elemental data for granites from Gaya. The dataset allows us to characterise these granitoids and help us understand their petrogenesis and put constraints on tectonic settings. 


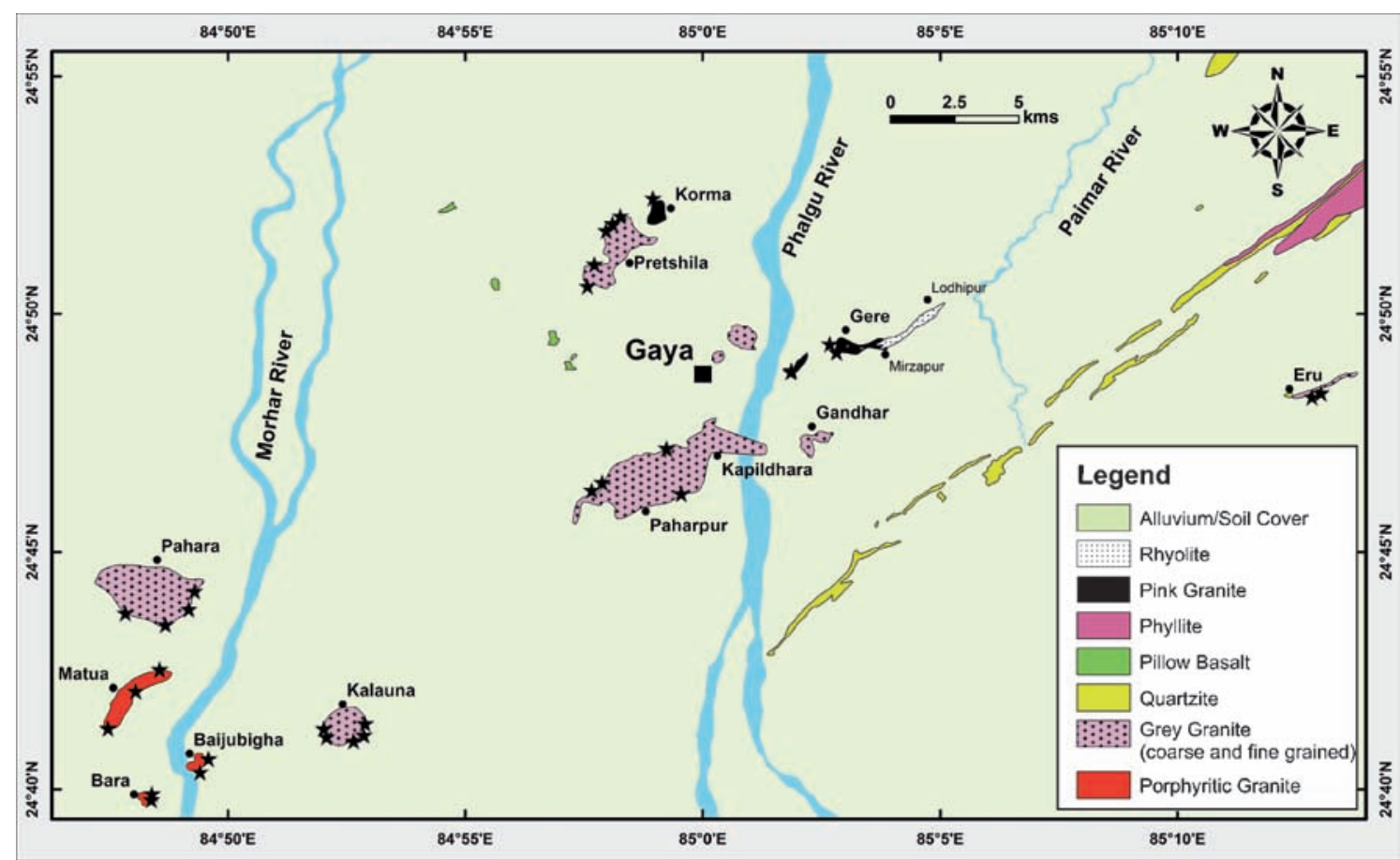

Figure 2. Detailed geological map of the study area showing various granitoid hillocks north of CGC in close proximity to Rajgir fold belt (modified after parts of Quadrangle Geological Map of $72 \mathrm{H}$ and $72 \mathrm{D}$ ). Locations of the samples presented in the study have been marked in the map with solid star $(\star)$.

\section{Geological background}

Chotanagpur Gneissic Complex (CGC), is an ENE-WSW trending high-grade terrain in the eastern part of Central Indian Tectonic Zone (CITZ) and covers an area of about $80,000 \mathrm{~km}^{2}$ (figure 1). It is bordered by the Singhbhum mobile belt in the south, the Quaternary Gangetic alluvium in the north and by the Rajmahal basalt in the northeast. In the west, CGC is separated from CITZ by younger Gondwana sediments. The terrain consists of mostly gneisses and migmatites with numerous metasedimentary enclaves of varying metamorphic grade which are in turn intruded by metabasic, anorthositic and granitic plutons, along with widespread but narrow belts of supracrustal enclaves, discontinuous boudin-type granulite belts and major parallel shear zones with a strike extension of about $500 \mathrm{~km}$ and a width of $200 \mathrm{~km}$ in $\mathrm{E}-\mathrm{W}$ direction. Younger mafic, ultramafic and alkaline (sodic and ultrapotassic) intrusives of early Tertiary age are also present (Dunn 1929; Ghose 1983, 1992; Mahadevan 2002; Mazumdar 1988; Sarkar 1988). The rocks of CGC are complexly deformed and metamorphosed showing varying degrees of metamorphism, exhibiting mainly amphibolite facies but ranges from greenschist (mostly in SE part of CGC) to granulite facies (central and eastern parts of CGC) (Bhattacharya 1976; Banerji 1991; Mahadevan 2002; Sharma 2009). Detailed structural studies have identified three phases of deformation in the CGC, giving rise to distinct fold patterns and related linear fabric (Chatterjee et al. 2008 and the references therein).

Based on the available geochronological data on the rocks of CGC, the terrain appeared to have experienced three tectono-thermal events during the Proterozoic. The first tectono-thermal event corresponds to the age group of 1.7-1.6 Ga while the second 1.2-1.0 Ga and third corresponds to 0.9 Ga (Chatterjee et al. 2008; Chatterjee and Ghose 2011; Karmakar et al. 2011; Sanyal and Sengupta 2012). In the wake of the complexity attained by the rocks of this terrain due to polyphase deformation, metamorphism, magmatism, metasomatism, lack of structural, geochemical, petrological and precise geochronological data, it is hard to ascertain the geological relationship between different litho-units and comment on the sequence of geological events in the region.

The study area, around Gaya, to the north of CGC, predominantly comprises of granitic plutons situated between the ENE and WSW trending Bihar Mica Belt (BMB) in the south and Barabar Anorthosite Complex in the north, whereas on the eastern margin, the Munger Group and the RajgirGaya Basin are situated (figure 1). The Munger Group and the Rajgir-Gaya Basin comprise of folded phyllite-quartzite sequences metamorphosed into greenschist facies with minor intrusive maficultramafic rocks and granitic bodies (Das 1967; 
Mazumdar 1988). The age and correlation of these rocks with other rocks of the same area are uncertain (Chatterjee and Ghose 2011). However, Das (1967) has correlated the Munger and Rajgir-Gaya sequences with Son-Narmada valley of central India, based on lithological and structural similarities. In the study area, out of three major sub-vertical $\mathrm{NE}-\mathrm{SW}$ trending faults present (figure 1), the NWdipping Monghyr-Saharsa Ridge Fault and SEdipping East Patna Fault taken together define a graben which probably represents a north-eastward extension of the Son-Narmada Graben of central India. Therefore, the granitic plutons along with the schist-phyllite-quartzite country rocks in the study area, lying on the northwestern side of the Monghyr-Saharsa Ridge Fault of the Rajgir-Gaya Basin may be correlated with the Mahakoshal Group of the Son-Narmada Graben (Chatterjee and Ghose 2011 and the references therein). Further, Chatterjee and Ghose (2011) have correlated these country rocks hosting the porphyritic granites around Gaya with Mahakoshal granitoids and supracrustals on the basis of $1697 \pm 17$ Ma monazite crystallisation age, and the absence of $<1.6 \mathrm{Ga}$ metamorphic imprint in the porphyritic granites.

\subsection{Field relationship}

The studied granitic plutons lie in parts of the Survey of India Toposheets No. 72 D/13, 72 $\mathrm{D} / 14$ and $72 \mathrm{H} / 1$ in Gaya district, Bihar (figure 2) and their exposures form circular to elliptical residual dome-like structures or inselbergs (figure 3a). Three types of granitic litho-units identified in the field on the basis of colour and texture are: (1) grey granite, (2) pink granite and (3) porphyritic granite. As the area is mostly covered with alluvium, the field relationship between litho-units are obscured and good exposures are found in pits of numerous quarries. The grey granite comprises two variants, the first one is leuco- to mesocratic, coarse-grained granites with an inequigranular texture. The second one is more widespread, leucocratic, medium-to-fine grained, equigranular granites which is seen intruding the first one (figure $3 \mathrm{~b}$ ). These two variants of grey granite are undifferentiated in the geological map and are observed around Gandhar, Kalauna, Kapildhara, Paharpur, Pahara and Pretshila (figure 2). These variants of granite are also seen intruding the anorthosites near Sapneri and Lachhubigha and volcano-sedimentary sequences near Bathani. Porphyritic granite occur as massive bodies, but at places, they are weakly foliated, sheared and are known to occur around Baijubigha, Bara and Matua (figure 2). At places, these granites are seen intruded by fine-grained grey granites (figure 3c), and are characterised by sub-rounded to tabular shaped phenocrysts of feldspars typically ranging in size from $2-4 \mathrm{~cm}$ in diameter (figure $3 \mathrm{e}$ ), but sometimes they can measure up to $5-6 \mathrm{~cm}$ (figure $3 \mathrm{~d}$ ). These phenocrysts constitute about $30-40 \%$ of the total mass and are randomly arranged, but at places, magmatic foliation aligned parallel to NE regional foliation trend is recorded. Occasionally rapakivi-like texture is observed in the feldspar phenocrysts. Pink granites are generally coarse to medium-grained, equigranular and homogeneous in nature (figure 3f) and are known to occur around Korma and Gere (figure 2). These granites display a clear inter-fingering contact with the rhyolite near Gere (figure 2). The $\mathrm{Rb}-\mathrm{Sr}$ wholerock dating of these rhyolites yields a crystallisation age of $1044 \pm 35$ Ma (Wanjari et al. 2012). Mafic magmatic enclaves (MME's) of variable sizes and angular to sub-rounded shapes are commonly seen in all the granitic litho-units. All these granitic plutons are interspersed with fine-grained aplitic or coarse-grained pegmatitic veins at places.

Wanjari et al. (2012) have reported pillow basalts and associated pyroclasts in the vicinity of Gaya along with rhyolite from Gere and Mirzapur and even suggested a back-arc basin like tectonic setting for the pillow basalts and mafic pyroclasts. Reports of occurrence of Bathani volcano-sedimentary sequences (BVS), northeast to our study area in juxtaposition to the Munger-Rajgir metasedimentary fold belt also adds emphasis to this study (Ahmad and Wanjari 2009; Saikia et al. 2014). This volcano-sedimentary sequence comprised of garnetmica schist, banded iron formation, chert bands, volcanic tuff, associated mafic pyroclasts and pillow basalts. Saikia et al. (2014) proposed a multistage and multi-source magmatism for BVS in an island-arc tectonic setting. It is thus imperative to envisage a viable geological model for the evolution of granitic rocks around Gaya and their interrelationships. Therefore, constraining the geochemical signatures of these granitic rocks will help us in understanding the geodynamic evolution of this area.

\section{Petrography}

\subsection{Grey granites}

Mineralogically, both types of grey granites are similar and shows holocrystalline, seriate to hypidiomorphic texture, and essentially consists of Qtz $(34$ vol. $\%)+\mathrm{Pl}(28$ vol. $\%)+\mathrm{Kfs}(24$ vol. $\%)+\mathrm{Hbl}$ (9 vol.\%)+Bt (4 vol.\%) (mineral symbols after Siivola and Schmid 2007) (figure 4a). Accessory minerals $(<1$ vol.\%) include primary $\mathrm{Ttn}+\mathrm{Ap}+$ $\mathrm{Zrn} \pm \mathrm{Aln}+\mathrm{Op}$, and secondary $\mathrm{Ep}+\mathrm{Chl}+\mathrm{Ser} \pm \mathrm{Ms}$. Quartz is present as strained grains and includes 
incipient sub-grains. Plagioclase, mostly unzoned and poikilitic, shows moderate alteration to sericitization and saussuritization in some places. Kfeldspar is mostly microcline and shows variable perthitic development with well-developed veins and flame to patchy perthites (figure 4b). Myrmekitic texture and micrographic texture is common. Euhedral to subhedral hornblende poikilitically encloses biotite, zircon, apatite and opaques, and coexist mostly in association with green to straw brown biotite (figure 4c). Biotite, at places, shows alteration to chlorite (figure $4 \mathrm{~d}$ ).

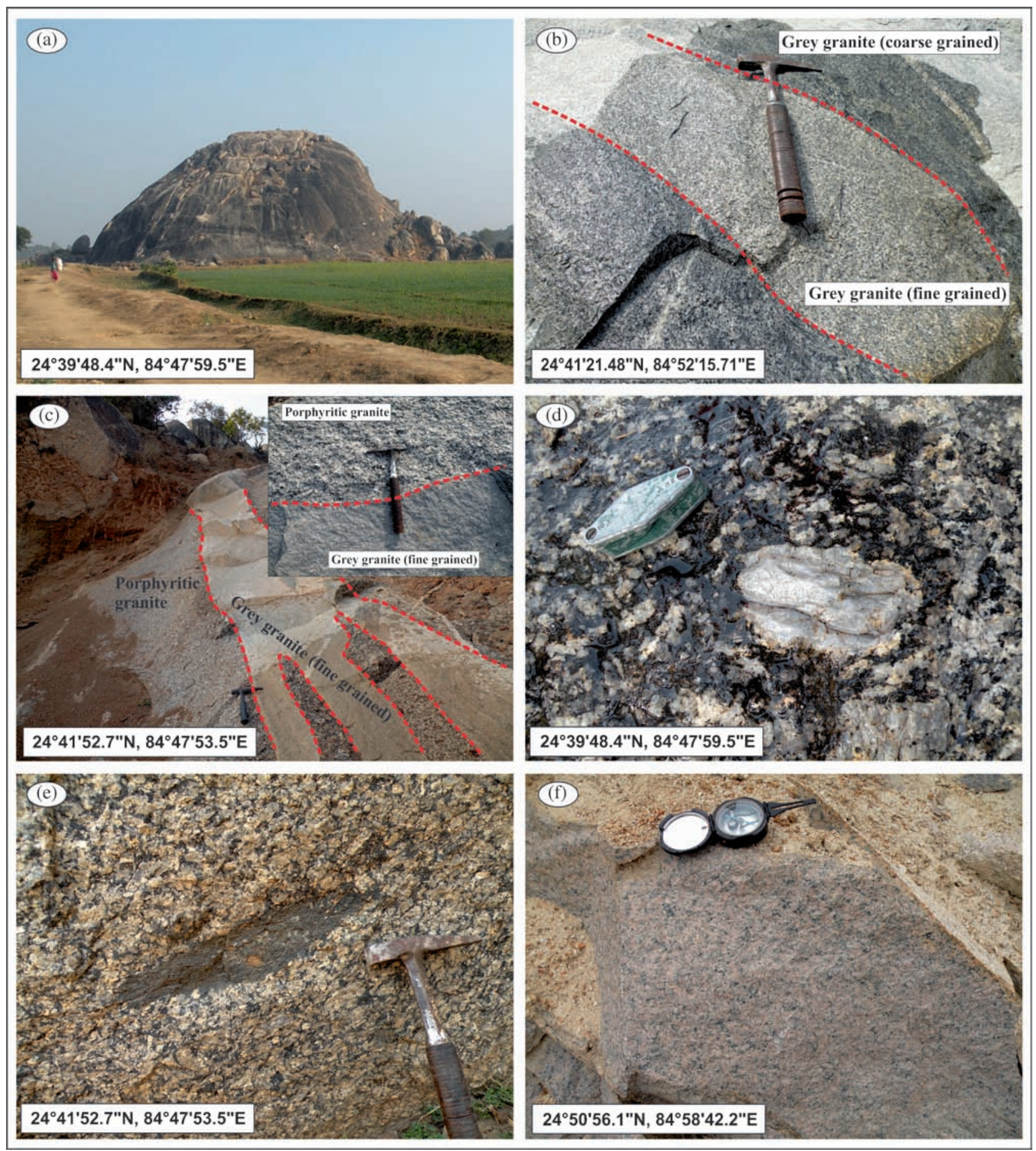

Figure 3. Field photographs of various granitic litho-units showing (a) exposure of granitic pluton near Bara, (b and c) crosscut relationship of fine-grained grey granite which intrudes both the coarse-grained grey granite and porphyritic granite. The inset in (c) shows close-up contact of fine grained grey granite and porphyritic granite, (d) phenocrysts of K-feldspar in porphyritic granite, (e) mafic microgranular enclave (MME) in porphyritic granite, and (f) outcrop of pink granite. 


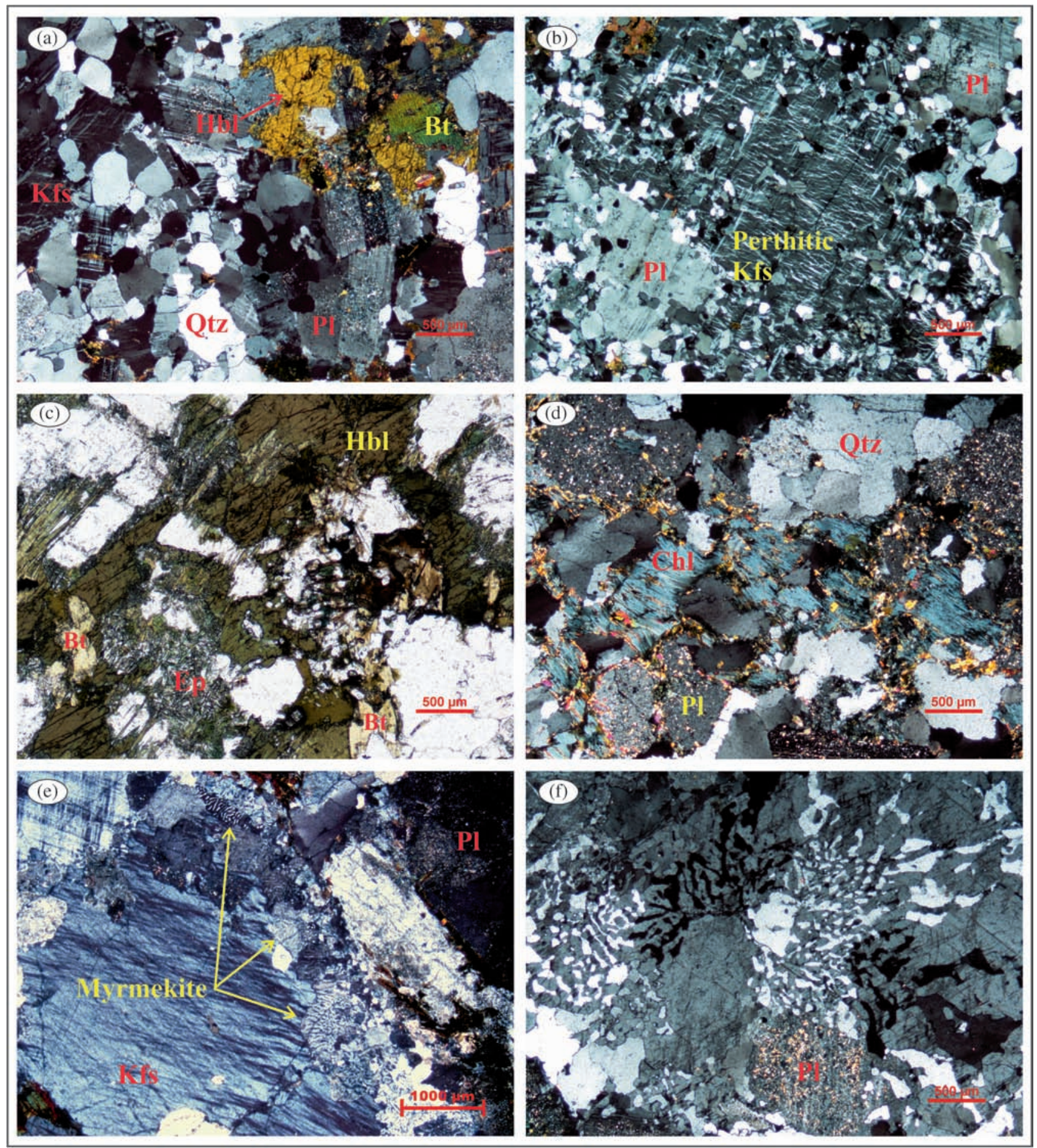

Figure 4. Photomicrographs of granitic litho-units showing (a) hypidiomorphic texture, minerals present are plagioclase, K-feldspar, quartz, hornblende and biotite (sample B-15), (b) perthitic intergrowth in K-feldspar with plagioclase and quartz grains (sample B-11), (c) hornblende and biotite grains in the matrix and associated epidote needles (sample B-12), (d) chlorite as alteration product of biotite is seen along with sericitised plagioclase and quartz grains (sample B-23), (e) myrmekite development at the boundary of K-feldspar (sample B-8), and (f) granophyric texture (sample B-10). (Qtz: quartz; Kfs: K-feldspar; Pl: plagioclase; Bt: biotite; Hbl: hornblende; Ep: epidote; Chl: chlorite).

\subsection{Pink granites}

The pink granites show hypidiomorphic texture, composed of Qtz (36 vol.\%), Kfs (29 vol.\%), Pl (25 vol.\%), Bt (7 vol.\%) and $\mathrm{Hbl}(2$ vol.\%). Accessory minerals ( $<1$ vol.\%) include only Zrn (commonly in biotite forming pleochroic halos $)+\mathrm{Ap}+\mathrm{Op} \pm \mathrm{Aln}$. $\mathrm{K}$-feldspar is more common than plagioclase, poikilitic and mainly shows simple twinning, but at places tartan twinning typical of microcline is 
also noticed. Exsolution and intergrowth textures like perthitic and granophyric are very common (figure 4f). These granites show microstructural deformation features, viz., strained quartz grains, kinked mica and deformation twins in plagioclase.

\subsection{Porphyritic granites}

The porphyritic granites are composed of $\mathrm{Kfs}$ (25 vol.\%) and Pl (10 vol.\%) phenocrysts set in a coarse grained matrix consisting of Qtz (26 vol.\%), $\mathrm{Pl}(19$ vol.\%), Kfs (10 vol.\%), Bt (8 vol.\%) and minor $\mathrm{Hbl}(<1$ vol.\%). They also contain accessory $(<1$ vol.\%) Zrn+Aln+Ap+Ttn+Op minerals. Phenocrysts of K-feldspar are mostly microcline and at places present with perthitic exsolution lamellas. Myrmekitic intergrowth is common along the grain boundaries of K-feldspar (figure 4e), and at places micrographic texture is present. The effects of shearing and deformation are evidenced by the presence of fine-grained recrystallised quartz, deformation twinning in feldspar, kinked mica, undulose extinction in quartz and development of a weak foliation defined by alignment of biotite.

\section{Analytical methods}

In this study, 32 samples (approx. 5-7 kg each) were collected but a total of 27 samples (15 grey granites, 5 pink granites and 7 porphyritic granites) with least alteration and deformation were analysed for major oxides, trace elements and rare earth elements (table 1). The average chemical index of alteration (CIA) value of all samples is 53 , which lies within the accepted 45-55 range for unweathered granites (Nesbitt and Young 1982). Loss on ignition (LOI) was determined by calcining the samples for 2 hours at $900^{\circ} \mathrm{C}$ in an electric furnace. Whole rock powders were analysed for major and most trace elements using WD-XRF (PANalytical AXIOUS PW4400/40 spectrometer) at the Department of Geology, University of Delhi, India. The accuracy of the analyses for the major elements is better than $1 \%$ for $\mathrm{SiO}_{2}$ and $2 \%$ for other major elements, $2-5 \%$ for minor elements and better than $10 \%$ for trace elements. Some trace and rare earth elements were analysed by ICP-MS (Perkin-Elmer Sciex ELAN DRC 6000) at the Institute Instrumentation Centre (IIC), Indian Institute of Technology Roorkee, India. Protocols for sample digestion and preparation of solutions for ICP-MS were that of Balaram et al. (1996). The international rock standards (USGS) used for calibrations for both WD-XRF and ICP-MS are JG-2 and DG-H. The precision of the data was expressed as the residual standard deviation (RSD), which in general for all the trace elements is much less than
$4 \%$. Individually, they are less than $2.5 \%$ for alkali and alkaline earth metals, whereas it is less than $2 \%$ for transition elements except Sc and Hf and within $3 \%$ for LREE and $4 \%$ for HREE (Bhattacharya et al. 2012).

\section{Geochemical characteristics}

On the basis of geochemical compositions and petrographic characteristics, grey granites (both, coarse and fine-grained variants) and pink granites have been classified together and herein named as GPG (20 samples) and the porphyritic granites have been named as PG (7 samples). Major and trace element data for all granites are listed in table 1.

\subsection{Major and trace elements}

The major and trace element distinctions between GPG and PG are subtle, but the real notable differences come in REE patterns. GPG and PG samples show similar abundances with high $\mathrm{SiO}_{2}$ $\left(\mathrm{GPG}_{\mathrm{avg}}=72.75\right.$ wt. $\% ; \mathrm{PG}_{\mathrm{avg}}=69$ wt. $\left.\%\right), \mathrm{Fe}_{2} \mathrm{O}_{3}{ }^{\mathrm{t}}$ $\left(\mathrm{GPG}_{\text {avg }}=3.75\right.$ wt. $\% ; \mathrm{PG}_{\text {avg }}=3.51$ wt. $\left.\%\right), \mathrm{Al}_{2} \mathrm{O}_{3}$, $\mathrm{Na}_{2} \mathrm{O}, \mathrm{K}_{2} \mathrm{O}$, low $\mathrm{MgO}$ and $\mathrm{CaO}$. However, on an average $\mathrm{PG}$ have higher $\mathrm{Al}_{2} \mathrm{O}_{3}, \mathrm{TiO}_{2}, \mathrm{MgO}$ and $\mathrm{P}_{2} \mathrm{O}_{5}$ content than GPG. Collectively, samples of both GPG and PG show high average contents of $\mathrm{Rb}(\sim 228 \mathrm{ppm})$, Ba ( 529 ppm), high field strength elements (HFSE) (Nb 21 ppm, $\mathrm{Zr} \sim$ $307 \mathrm{ppm}, \mathrm{Y} \sim 48 \mathrm{ppm}$, Th $25 \mathrm{ppm}$ and $\mathrm{U} \sim 3 \mathrm{ppm})$ and depletion in $\mathrm{Sr}(\sim 56 \mathrm{ppm})$. All samples of GPG and PG have normative quartz, corundum and hypersthene. In the normative QAP classification diagram (figure 5a), all samples plot in the monzogranite field except sample B-23 which lies in the granodiorite field. On the other hand, on the Ab-An-Or diagram of O'connor (1965) modified after Barker (1979) (figure not shown), all samples plot in granite field. The samples of GPG are slightly metaluminous to peraluminous $(\mathrm{A} / \mathrm{CNK}=0.97-1.36)$ while those of $\mathrm{PG}$ are peraluminous $(\mathrm{A} / \mathrm{CNK}=1.07-1.21)$ (figure $5 \mathrm{~b})$. According to the classification of Frost et al. (2001), all samples belong to the ferroan granite on $\mathrm{Fe}^{*}\left[\mathrm{FeO}^{\mathrm{t}} /\left(\mathrm{FeO}^{\mathrm{t}}+\mathrm{MgO}\right)\right]$ vs. $\mathrm{SiO}_{2}$ diagram (figure $5 \mathrm{c})$, whereas on the MALI $\left(\mathrm{Na}_{2} \mathrm{O}+\mathrm{K}_{2} \mathrm{O}-\mathrm{CaO}\right)$ vs. $\mathrm{SiO}_{2}$ (figure $5 \mathrm{~d}$ ), samples of GPG mostly plot in the calc-alkalic and those of PG plot in alkaliccalcic fields. These observations are consistent with the chemical characteristics of worldwide A-type granites. Although the granites have high Fe*, interestingly they are K-rich and their average $\mathrm{K}_{2} \mathrm{O} / \mathrm{Na}_{2} \mathrm{O} \sim 1.64$ ratios are quite high. This indicates that the granites in the study area are roughly similar to the peraluminous A-type granites. 
Table 1. Representative whole rock compositions of granites from Gaya.

\begin{tabular}{|c|c|c|c|c|c|c|c|c|c|}
\hline $\begin{array}{l}\text { Sample } \\
\text { Rock type }\end{array}$ & $\begin{array}{l}\text { B-02 } \\
\text { FGG }\end{array}$ & $\begin{array}{l}\text { B-05 } \\
\text { FGG }\end{array}$ & $\begin{array}{l}\text { B-13 } \\
\text { FGG }\end{array}$ & $\begin{array}{l}\text { B-14 } \\
\text { FGG }\end{array}$ & $\begin{array}{l}\text { B-15 } \\
\text { FGG }\end{array}$ & $\begin{array}{l}\text { B-19 } \\
\text { FGG }\end{array}$ & $\begin{array}{l}\text { B-21 } \\
\text { FGG }\end{array}$ & $\begin{array}{l}\text { B-22 } \\
\text { FGG }\end{array}$ & $\begin{array}{l}\text { B-23 } \\
\text { FGG }\end{array}$ \\
\hline \multicolumn{10}{|c|}{ Major oxides (wt.\%) } \\
\hline $\mathrm{SiO}_{2}$ & 70.98 & 76.03 & 71.93 & 73.07 & 68.02 & 77.06 & 75.60 & 74.97 & 64.62 \\
\hline $\mathrm{Al}_{2} \mathrm{O}_{3}$ & 13.18 & 13.83 & 13.86 & 13.57 & 13.75 & 14.51 & 13.90 & 14.14 & 15.61 \\
\hline $\mathrm{Na}_{2} \mathrm{O}$ & 2.95 & 3.30 & 2.69 & 2.88 & 3.15 & 3.17 & 2.90 & 2.98 & 3.59 \\
\hline $\mathrm{MgO}$ & 0.16 & 0.03 & 0.05 & 0.11 & 0.16 & 0.06 & 0.07 & 0.06 & 1.03 \\
\hline $\mathrm{MnO}$ & 0.09 & 0.05 & 0.08 & 0.09 & 0.12 & 0.05 & 0.04 & 0.04 & 0.04 \\
\hline $\mathrm{Fe}_{2} \mathrm{O}_{3}{ }^{\mathrm{t}}$ & 5.08 & 2.61 & 4.67 & 4.63 & 5.88 & 2.74 & 2.45 & 2.41 & 6.07 \\
\hline $\mathrm{K}_{2} \mathrm{O}$ & 4.62 & 4.66 & 4.81 & 4.67 & 4.62 & 5.03 & 5.46 & 5.34 & 2.53 \\
\hline $\mathrm{CaO}$ & 1.72 & 0.96 & 1.69 & 1.43 & 2.19 & 1.06 & 0.99 & 1.02 & 2.07 \\
\hline $\mathrm{TiO}_{2}$ & 0.34 & 0.20 & 0.35 & 0.31 & 0.40 & 0.21 & 0.20 & 0.20 & 0.52 \\
\hline $\mathrm{P}_{2} \mathrm{O}_{5}$ & 0.06 & 0.02 & 0.03 & 0.03 & 0.06 & 0.03 & 0.03 & 0.03 & 0.05 \\
\hline LOI & 0.83 & 0.51 & 0.76 & 0.72 & 0.68 & 0.55 & 0.52 & 0.45 & 1.95 \\
\hline Total & 100.00 & 102.20 & 100.92 & 101.50 & 99.03 & 104.47 & 102.15 & 101.63 & 98.06 \\
\hline CIA & 50 & 53 & 52 & 52 & 49 & 54 & 53 & 53 & 56 \\
\hline $\mathrm{Fe}^{*}$ & 0.97 & 0.99 & 0.99 & 0.97 & 0.97 & 0.97 & 0.97 & 0.97 & 0.84 \\
\hline MALI & 5.86 & 7.00 & 5.82 & 6.11 & 5.58 & 7.15 & 7.36 & 7.30 & 4.05 \\
\hline \multicolumn{10}{|c|}{ Trace elements (ppm) } \\
\hline $\mathrm{Rb}$ & 204 & 240 & 178 & 209 & 241 & 243 & 226 & 239 & 170 \\
\hline $\mathrm{Ba}$ & 750 & 649 & 678 & 623 & 563 & 522 & 516 & 737 & 561 \\
\hline Th & 8 & 16 & 21 & 23 & 21 & 19 & 19 & 8 & 25 \\
\hline $\mathrm{U}$ & 3 & 2 & 2 & 4 & 5 & 2 & 2 & 1 & 9 \\
\hline $\mathrm{Nb}$ & 20 & 18 & 16 & 23 & 29 & 18 & 17 & 18 & 25 \\
\hline $\mathrm{Pb}$ & 29 & 24 & 24 & 27 & 24 & 27 & 22 & 22 & 19 \\
\hline $\mathrm{Sr}$ & 87 & 34 & 51 & 61 & 70 & 35 & 36 & 41 & 66 \\
\hline $\mathrm{Zr}$ & 294 & 328 & 315 & 360 & 404 & 344 & 324 & 300 & 509 \\
\hline $\mathrm{Hf}$ & 7 & 6 & 6 & 6 & 6 & 6 & 6 & 6 & 3 \\
\hline $\mathrm{Y}$ & 52 & 54 & 46 & 57 & 63 & 61 & 57 & 52 & 70 \\
\hline $\mathrm{Ni}$ & 6 & 21 & 24 & 15 & 5 & 10 & 24 & 15 & 19 \\
\hline $\mathrm{Cr}$ & 651 & 733 & 522 & 779 & 580 & 726 & 557 & 518 & 419 \\
\hline $\mathrm{V}$ & 11 & 5 & 10 & 9 & 16 & 8 & 6 & 2 & 34 \\
\hline Co & 8 & 4 & 8 & 5 & 4 & 6 & 6 & 5 & 0 \\
\hline $\mathrm{Sc}$ & 21 & 2 & 1 & 12 & 15 & 7 & 4 & 9 & 0 \\
\hline $\mathrm{Cu}$ & 18 & 7 & 36 & 6 & 10 & 7 & 3 & 1 & 0 \\
\hline $\mathrm{Ga}$ & 22 & 22 & 23 & 23 & 23 & 23 & 23 & 22 & 25 \\
\hline $\mathrm{Zn}$ & 94 & 82 & 78 & 108 & 142 & 85 & 62 & 78 & 27 \\
\hline $\mathrm{La}$ & 30.70 & 26.21 & 20.56 & 27.24 & 24.82 & 24.58 & 24.22 & 22.93 & 33.63 \\
\hline $\mathrm{Ce}$ & 69.27 & 58.80 & 46.55 & 61.06 & 56.02 & 54.49 & 55.31 & 52.54 & 75.64 \\
\hline $\mathrm{Nd}$ & 39.74 & 33.54 & 26.99 & 35.31 & 32.61 & 30.93 & 32.10 & 30.68 & 43.77 \\
\hline $\mathrm{Sm}$ & 9.02 & 7.53 & 6.24 & 8.13 & 7.60 & 6.88 & 7.43 & 7.19 & 10.09 \\
\hline $\mathrm{Eu}$ & 2.27 & 1.49 & 1.45 & 1.93 & 2.03 & 1.29 & 1.38 & 1.64 & 2.07 \\
\hline Gd & 10.07 & 8.38 & 7.17 & 9.43 & 8.82 & 7.92 & 8.39 & 8.15 & 11.13 \\
\hline $\mathrm{Tb}$ & 1.55 & 1.27 & 1.11 & 1.47 & 1.37 & 1.21 & 1.29 & 1.23 & 1.69 \\
\hline Dy & 9.89 & 7.82 & 7.04 & 9.49 & 9.11 & 7.69 & 8.09 & 7.53 & 10.38 \\
\hline Но & 1.95 & 1.51 & 1.39 & 1.87 & 1.85 & 1.51 & 1.59 & 1.47 & 2.02 \\
\hline Er & 5.01 & 3.79 & 3.56 & 4.80 & 4.94 & 3.84 & 4.04 & 3.70 & 5.07 \\
\hline $\mathrm{Yb}$ & 5.31 & 3.65 & 3.64 & 5.02 & 5.92 & 3.86 & 4.04 & 3.60 & 5.69 \\
\hline $\mathrm{Lu}$ & 0.83 & 0.54 & 0.55 & 0.76 & 0.92 & 0.58 & 0.62 & 0.55 & 0.82 \\
\hline $\mathrm{Eu} / \mathrm{Eu}^{*}$ & 0.73 & 0.57 & 0.66 & 0.67 & 0.76 & 0.53 & 0.53 & 0.65 & 0.60 \\
\hline$(\mathrm{La} / \mathrm{Lu})_{\mathrm{N}}$ & 3.86 & 5.02 & 3.85 & 3.70 & 2.79 & 4.44 & 4.07 & 4.36 & 4.25 \\
\hline $\mathrm{T}_{\mathrm{Zr}}\left({ }^{\circ} \mathrm{C}\right)$ & 841.61 & 868.71 & 857.10 & 872.21 & 862.90 & 874.62 & 865.26 & 858.66 & 914.99 \\
\hline
\end{tabular}


Table 1. (Continued.)

\begin{tabular}{|c|c|c|c|c|c|c|c|c|c|}
\hline $\begin{array}{l}\text { Sample } \\
\text { Rock type }\end{array}$ & $\begin{array}{l}\text { B-11 } \\
\text { CGG }\end{array}$ & $\begin{array}{l}\text { B-12 } \\
\text { CGG }\end{array}$ & $\begin{array}{l}\text { B-24 } \\
\text { CGG }\end{array}$ & $\begin{array}{l}\text { B-25 } \\
\text { CGG }\end{array}$ & $\begin{array}{l}\text { B-31 } \\
\text { CGG }\end{array}$ & $\begin{array}{l}\text { B-32 } \\
\text { CGG }\end{array}$ & $\begin{array}{l}\text { B-03 } \\
\text { PG }\end{array}$ & $\begin{array}{l}\text { B-10 } \\
\text { PG }\end{array}$ & $\begin{array}{c}\mathrm{B}-27 \\
\mathrm{PG}\end{array}$ \\
\hline \multicolumn{10}{|c|}{ Major oxides (wt.\%) } \\
\hline $\mathrm{SiO}_{2}$ & 74.97 & 65.45 & 70.79 & 72.40 & 75.18 & 72.40 & 76.19 & 73.50 & 73.98 \\
\hline $\mathrm{Al}_{2} \mathrm{O}_{3}$ & 13.89 & 13.98 & 13.88 & 13.99 & 13.75 & 14.11 & 13.45 & 14.07 & 14.39 \\
\hline $\mathrm{Na}_{2} \mathrm{O}$ & 3.03 & 2.95 & 2.87 & 2.28 & 3.44 & 2.88 & 3.49 & 3.14 & 3.25 \\
\hline $\mathrm{MgO}$ & 0.17 & 0.27 & 0.72 & 0.75 & 0.17 & 0.33 & 0.35 & 0.13 & 0.20 \\
\hline $\mathrm{MnO}$ & 0.05 & 0.14 & 0.01 & 0.03 & 0.02 & 0.03 & 0.01 & 0.04 & 0.06 \\
\hline $\mathrm{Fe}_{2} \mathrm{O}_{3}{ }^{\mathrm{t}}$ & 3.27 & 7.82 & 3.87 & 3.84 & 2.11 & 2.81 & 2.78 & 3.01 & 3.18 \\
\hline $\mathrm{K}_{2} \mathrm{O}$ & 4.94 & 3.57 & 4.67 & 5.45 & 4.32 & 5.20 & 4.71 & 5.14 & 4.94 \\
\hline $\mathrm{CaO}$ & 1.21 & 2.68 & 0.78 & 0.37 & 1.04 & 0.94 & 0.49 & 1.08 & 1.22 \\
\hline $\mathrm{TiO}_{2}$ & 0.24 & 0.54 & 0.26 & 0.26 & 0.14 & 0.23 & 0.21 & 0.22 & 0.20 \\
\hline $\mathrm{P}_{2} \mathrm{O}_{5}$ & 0.03 & 0.09 & 0.03 & 0.03 & 0.02 & 0.04 & 0.02 & 0.03 & 0.02 \\
\hline LOI & 0.64 & 0.87 & 1.08 & 1.09 & 1.00 & 0.79 & 0.40 & 0.82 & 1.12 \\
\hline Total & 102.45 & 98.35 & 98.95 & 100.49 & 101.19 & 99.77 & 102.10 & 101.17 & 102.54 \\
\hline CIA & 53 & 51 & 55 & 58 & 53 & 54 & 53 & 53 & 53 \\
\hline $\mathrm{Fe}^{*}$ & 0.94 & 0.96 & 0.83 & 0.82 & 0.92 & 0.89 & 0.88 & 0.96 & 0.93 \\
\hline MALI & 6.76 & 3.84 & 6.77 & 7.37 & 6.72 & 7.15 & 7.71 & 7.19 & 6.96 \\
\hline \multicolumn{10}{|c|}{ Trace elements (ppm) } \\
\hline $\mathrm{Rb}$ & 238 & 174 & 210 & 166 & 185 & 221 & 102 & 279 & 217 \\
\hline $\mathrm{Ba}$ & 706 & 456 & 386 & 540 & 143 & 272 & 412 & 710 & 662 \\
\hline $\mathrm{Th}$ & 24 & 19 & 21 & 24 & 42 & 40 & 22 & 20 & 25 \\
\hline $\mathrm{U}$ & 4 & 3 & 3 & 2 & 8 & 7 & 1 & 4 & 4 \\
\hline $\mathrm{Nb}$ & 22 & 26 & 23 & 20 & 17 & 16 & 16 & 23 & 23 \\
\hline $\mathrm{Pb}$ & 26 & 23 & 10 & 12 & 35 & 33 & 12 & 23 & 29 \\
\hline $\mathrm{Sr}$ & 48 & 90 & 37 & 27 & 23 & 36 & 31 & 34 & 37 \\
\hline $\mathrm{Zr}$ & 318 & 393 & 315 & 335 & 152 & 169 & 391 & 301 & 350 \\
\hline $\mathrm{Hf}$ & 7 & 5 & 6 & 7 & 7 & 10 & 6 & 7 & 10 \\
\hline $\mathrm{Y}$ & 58 & 44 & 47 & 32 & 77 & 73 & 31 & 66 & 62 \\
\hline $\mathrm{Ni}$ & 20 & 14 & 17 & 22 & 7 & 24 & 21 & 21 & 14 \\
\hline $\mathrm{Cr}$ & 604 & 386 & 562 & 407 & 881 & 760 & 828 & 677 & 486 \\
\hline $\mathrm{V}$ & 15 & 20 & 12 & 15 & 6 & 19 & 11 & 10 & 8 \\
\hline $\mathrm{Co}$ & 4 & 4 & 6 & 5 & 4 & 8 & 4 & 5 & 8 \\
\hline $\mathrm{Sc}$ & 11 & 9 & 9 & 3 & 2 & 0 & 9 & 23 & 14 \\
\hline $\mathrm{Cu}$ & 2 & 39 & 0 & 44 & 1 & 5 & 10 & 15 & 1 \\
\hline $\mathrm{Ga}$ & 21 & 23 & 21 & 18 & 21 & 20 & 23 & 23 & 23 \\
\hline $\mathrm{Zn}$ & 85 & 132 & 17 & 13 & 24 & 39 & 13 & 58 & 89 \\
\hline $\mathrm{La}$ & 30.46 & 21.79 & 28.81 & 26.06 & 24.99 & 22.63 & 34.83 & 27.50 & 27.66 \\
\hline $\mathrm{Ce}$ & 67.04 & 50.65 & 62.61 & 56.64 & 51.02 & 47.04 & 74.97 & 60.70 & 61.27 \\
\hline $\mathrm{Nd}$ & 37.40 & 30.25 & 33.84 & 30.21 & 25.83 & 23.66 & 39.19 & 34.22 & 34.83 \\
\hline $\mathrm{Sm}$ & 8.04 & 7.37 & 6.80 & 5.89 & 4.41 & 3.97 & 7.28 & 7.51 & 7.77 \\
\hline $\mathrm{Eu}$ & 1.67 & 2.31 & 1.35 & 1.70 & 0.24 & 0.41 & 1.49 & 1.50 & 1.59 \\
\hline $\mathrm{Gd}$ & 9.14 & 8.38 & 7.58 & 6.58 & 5.44 & 4.82 & 8.20 & 8.47 & 8.87 \\
\hline $\mathrm{Tb}$ & 1.35 & 1.33 & 1.06 & 0.82 & 0.79 & 0.71 & 1.11 & 1.30 & 1.37 \\
\hline Dy & 8.54 & 8.40 & 6.16 & 4.44 & 5.16 & 4.69 & 6.37 & 8.44 & 8.89 \\
\hline Ho & 1.69 & 1.67 & 1.21 & 0.85 & 1.10 & 1.00 & 1.23 & 1.68 & 1.79 \\
\hline $\mathrm{Er}$ & 4.32 & 4.32 & 3.08 & 2.08 & 3.11 & 2.81 & 3.09 & 4.35 & 4.70 \\
\hline $\mathrm{Yb}$ & 4.50 & 4.71 & 3.19 & 2.15 & 3.45 & 3.33 & 3.13 & 4.64 & 5.04 \\
\hline $\mathrm{Lu}$ & 0.69 & 0.74 & 0.51 & 0.36 & 0.52 & 0.49 & 0.49 & 0.71 & 0.77 \\
\hline $\mathrm{Eu} / \mathrm{Eu}^{*}$ & 0.59 & 0.90 & 0.57 & 0.83 & 0.15 & 0.28 & 0.59 & 0.57 & 0.58 \\
\hline$(\mathrm{La} / \mathrm{Lu})_{\mathrm{N}}$ & 4.59 & 3.07 & 5.90 & 7.53 & 4.95 & 4.81 & 7.32 & 4.04 & 3.74 \\
\hline $\mathrm{T}_{\mathrm{Zr}}\left({ }^{\circ} \mathrm{C}\right)$ & 862.32 & 864.98 & 871.01 & 887.22 & 795.69 & 806.22 & 888.20 & 855.64 & 870.95 \\
\hline
\end{tabular}


Table 1. (Continued.)

\begin{tabular}{|c|c|c|c|c|c|c|c|c|c|}
\hline $\begin{array}{l}\text { Sample } \\
\text { Rock type }\end{array}$ & $\begin{array}{c}\text { B-28 } \\
\text { PG }\end{array}$ & $\begin{array}{c}\mathrm{B}-30 \\
\mathrm{PG}\end{array}$ & $\begin{array}{l}\mathrm{B}-06 \\
\mathrm{PG}^{*}\end{array}$ & $\begin{array}{l}\mathrm{B}-07 \\
\mathrm{PG}^{*}\end{array}$ & $\begin{array}{l}\mathrm{B}-08 \\
\mathrm{PG}^{*}\end{array}$ & $\begin{array}{l}\mathrm{B}-09 \\
\mathrm{PG}^{*}\end{array}$ & $\begin{array}{l}\mathrm{B}-16 \\
\mathrm{PG}^{*}\end{array}$ & $\begin{array}{l}\mathrm{B}-17 \\
\mathrm{PG}^{*}\end{array}$ & $\begin{array}{l}\text { B-18 } \\
\text { PG* }^{*}\end{array}$ \\
\hline \multicolumn{10}{|c|}{ Major oxides (wt.\%) } \\
\hline $\mathrm{SiO}_{2}$ & 70.02 & 77.85 & 69.38 & 77.09 & 62.67 & 65.79 & 68.37 & 69.95 & 69.78 \\
\hline $\mathrm{Al}_{2} \mathrm{O}_{3}$ & 14.57 & 14.13 & 15.65 & 13.83 & 15.30 & 14.96 & 14.68 & 15.37 & 15.70 \\
\hline $\mathrm{Na}_{2} \mathrm{O}$ & 3.20 & 3.09 & 2.84 & 2.46 & 2.76 & 2.95 & 2.94 & 2.42 & 2.73 \\
\hline $\mathrm{MgO}$ & 0.71 & 0.11 & 0.64 & 0.14 & 1.06 & 1.02 & 0.71 & 0.29 & 0.51 \\
\hline $\mathrm{MnO}$ & 0.03 & 0.05 & 0.03 & 0.02 & 0.07 & 0.07 & 0.05 & 0.02 & 0.03 \\
\hline $\mathrm{Fe}_{2} \mathrm{O}_{3}{ }^{\mathrm{t}}$ & 4.31 & 1.55 & 3.61 & 1.36 & 5.56 & 5.20 & 4.10 & 1.78 & 2.96 \\
\hline $\mathrm{K}_{2} \mathrm{O}$ & 3.65 & 5.57 & 5.12 & 5.45 & 4.83 & 4.97 & 4.46 & 6.85 & 5.93 \\
\hline $\mathrm{CaO}$ & 1.97 & 0.58 & 1.90 & 0.80 & 2.14 & 2.09 & 2.03 & 1.03 & 1.39 \\
\hline $\mathrm{TiO}_{2}$ & 0.51 & 0.14 & 0.43 & 0.16 & 0.71 & 0.66 & 0.50 & 0.18 & 0.31 \\
\hline $\mathrm{P}_{2} \mathrm{O}_{5}$ & 0.08 & 0.02 & 0.13 & 0.03 & 0.21 & 0.21 & 0.14 & 0.11 & 0.11 \\
\hline LOI & 1.34 & 1.09 & 1.06 & 0.53 & 0.83 & 0.75 & 0.88 & 0.59 & 0.77 \\
\hline Total & 100.41 & 104.16 & 100.78 & 101.86 & 96.14 & 98.67 & 98.85 & 98.59 & 100.23 \\
\hline CIA & 53 & 54 & 53 & 55 & 53 & 52 & 52 & 54 & 54 \\
\hline $\mathrm{Fe}^{*}$ & 0.84 & 0.93 & 0.83 & 0.90 & 0.82 & 0.82 & 0.84 & 0.85 & 0.84 \\
\hline MALI & 4.88 & 8.08 & 6.06 & 7.10 & 5.45 & 5.83 & 5.36 & 8.24 & 7.27 \\
\hline \multicolumn{10}{|c|}{ Trace elements (ppm) } \\
\hline $\mathrm{Rb}$ & 183 & 205 & 227 & 234 & 252 & 218 & 229 & 278 & 259 \\
\hline $\mathrm{Ba}$ & 435 & 321 & 583 & 256 & 615 & 902 & 318 & 477 & 610 \\
\hline $\mathrm{Th}$ & 13 & 33 & 35 & 11 & 35 & 11 & 21 & 6 & 31 \\
\hline $\mathrm{U}$ & 4 & 7 & 2 & 2 & 1 & 2 & 2 & 1 & 3 \\
\hline $\mathrm{Nb}$ & 22 & 28 & 17 & 8 & 30 & 27 & 24 & 13 & 21 \\
\hline $\mathrm{Pb}$ & 20 & 21 & 27 & 21 & 15 & 18 & 20 & 21 & 32 \\
\hline $\mathrm{Sr}$ & 65 & 21 & 113 & 75 & 89 & 98 & 69 & 72 & 90 \\
\hline $\mathrm{Zr}$ & 378 & 198 & 335 & 145 & 351 & 243 & 293 & 186 & 253 \\
\hline Hf & 5 & 7 & 8 & 5 & 4 & 3 & 5 & 6 & 7 \\
\hline $\mathrm{Y}$ & 44 & 65 & 19 & 19 & 42 & 33 & 28 & 29 & 24 \\
\hline $\mathrm{Ni}$ & 18 & 15 & 14 & 7 & 4 & 14 & 8 & 4 & 10 \\
\hline $\mathrm{Cr}$ & 590 & 1251 & 608 & 752 & 420 & 444 & 576 & 555 & 530 \\
\hline $\mathrm{V}$ & 31 & 9 & 30 & 6 & 46 & 50 & 33 & 17 & 19 \\
\hline $\mathrm{Co}$ & 8 & 5 & 9 & 6 & 10 & 9 & 7 & 9 & 4 \\
\hline $\mathrm{Sc}$ & 4 & 10 & 0 & 6 & 13 & 11 & 12 & 13 & 12 \\
\hline $\mathrm{Cu}$ & 20 & 3 & 23 & 1 & 11 & 11 & 6 & 0 & 0 \\
\hline $\mathrm{Ga}$ & 21 & 22 & 23 & 19 & 23 & 20 & 24 & 21 & 20 \\
\hline $\mathrm{Zn}$ & 37 & 9 & 55 & 17 & 75 & 71 & 58 & 26 & 49 \\
\hline $\mathrm{La}$ & 22.85 & 28.24 & 44.79 & 34.24 & 54.53 & 18.69 & 30.06 & 12.53 & 31.87 \\
\hline $\mathrm{Ce}$ & 48.88 & 61.98 & 93.52 & 68.01 & 111.04 & 41.59 & 61.11 & 27.05 & 66.86 \\
\hline $\mathrm{Nd}$ & 26.52 & 34.37 & 44.15 & 30.78 & 53.69 & 22.74 & 28.97 & 14.04 & 32.61 \\
\hline $\mathrm{Sm}$ & 5.38 & 7.30 & 6.02 & 3.51 & 7.97 & 4.69 & 4.01 & 2.56 & 4.98 \\
\hline $\mathrm{Eu}$ & 1.18 & 0.60 & 1.26 & 1.06 & 1.31 & 1.39 & 0.88 & 0.89 & 1.19 \\
\hline Gd & 6.45 & 8.66 & 6.10 & 3.87 & 8.71 & 5.05 & 4.72 & 2.60 & 4.92 \\
\hline $\mathrm{Tb}$ & 1.00 & 1.35 & 0.48 & 0.32 & 1.00 & 0.69 & 0.50 & 0.29 & 0.42 \\
\hline Dy & 6.50 & 8.77 & 1.46 & 1.19 & 4.91 & 3.90 & 2.30 & 1.46 & 1.45 \\
\hline Ho & 1.31 & 1.77 & 0.28 & 0.23 & 0.90 & 0.75 & 0.43 & 0.28 & 0.26 \\
\hline Er & 3.42 & 4.65 & 0.70 & 0.57 & 2.08 & 1.87 & 1.04 & 0.67 & 0.61 \\
\hline $\mathrm{Yb}$ & 3.62 & 5.08 & 0.52 & 0.49 & 1.42 & 1.85 & 0.86 & 0.64 & 0.46 \\
\hline $\mathrm{Lu}$ & 0.53 & 0.76 & 0.10 & 0.08 & 0.21 & 0.28 & 0.14 & 0.10 & 0.08 \\
\hline $\mathrm{Eu} / \mathrm{Eu}^{*}$ & 0.61 & 0.23 & 0.63 & 0.88 & 0.48 & 0.87 & 0.62 & 1.05 & 0.74 \\
\hline$(\mathrm{La} / \mathrm{Lu})_{\mathrm{N}}$ & 4.45 & 3.86 & 44.92 & 44.42 & 27.61 & 6.82 & 22.13 & 12.94 & 40.34 \\
\hline $\mathrm{T}_{\mathrm{Zr}}\left({ }^{\circ} \mathrm{C}\right)$ & 877.70 & 823.06 & 864.19 & 799.69 & 859.81 & 821.12 & 846.88 & 811.75 & 839.31 \\
\hline
\end{tabular}

FGG: Fine grained grey granite; CGG: Coarse grained grey granite; PG: Pink granite; PG*: Porphyritic granite. 

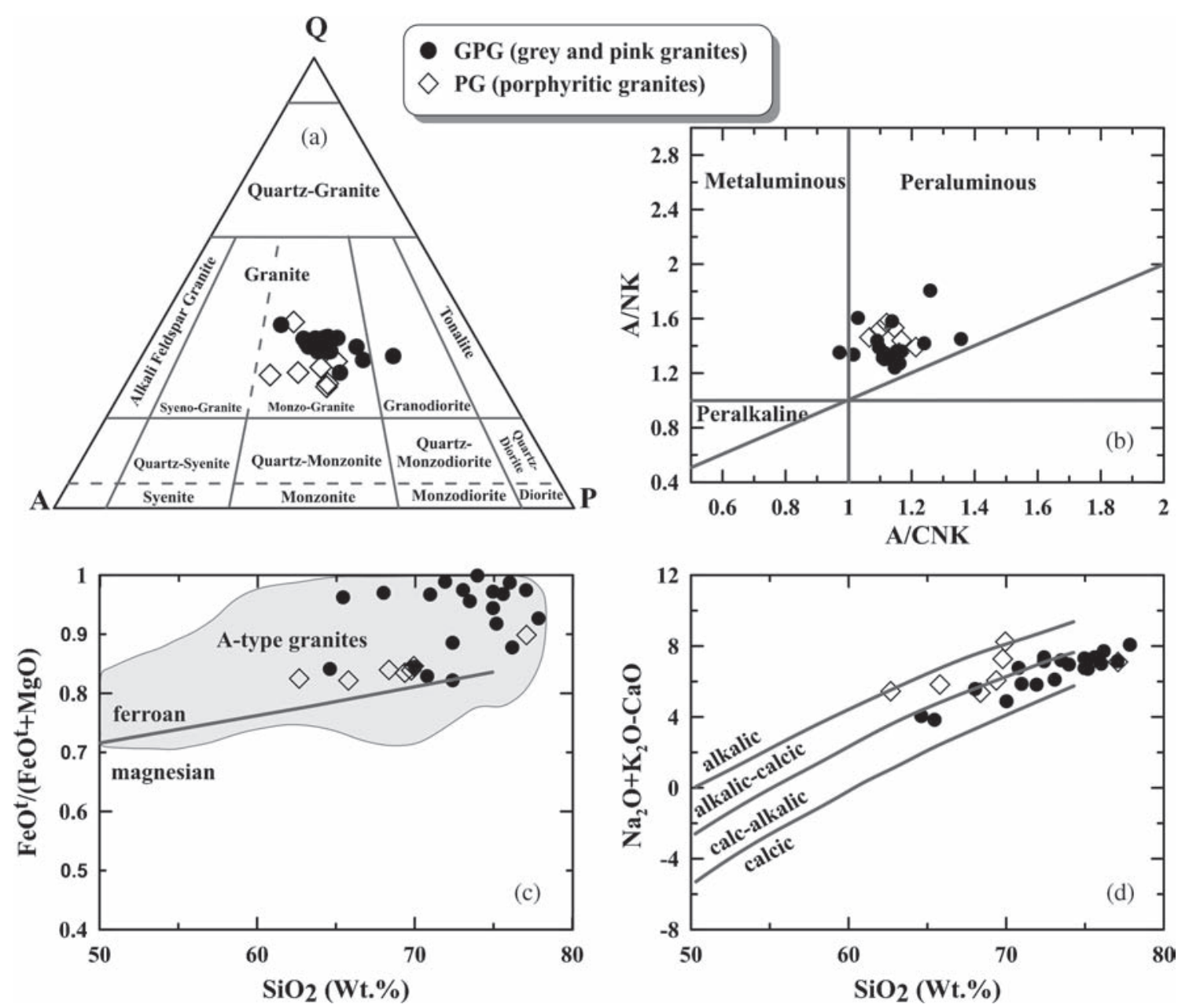

Figure 5. (a) Q-A-P normative plot after Le Maitre et al. (2002) showing granitic composition for the samples, (b) ACNK $\left(\right.$ molar $\left.\mathrm{Al}_{2} \mathrm{O}_{3} /\left(\mathrm{CaO}+\mathrm{Na}_{2} \mathrm{O}+\mathrm{K}_{2} \mathrm{O}\right)\right)$ vs. A/NK (molar $\mathrm{Al}_{2} \mathrm{O}_{3} /\left(\mathrm{Na}_{2} \mathrm{O}+\mathrm{K}_{2} \mathrm{O}\right)$ ) diagram (field after Maniar and Piccoli 1989) showing chemical composition of GPG and PG in terms of alumina saturation, (c) $\mathrm{Fe}^{*}\left[\mathrm{FeO}^{\mathrm{t}} /\left(\mathrm{FeO}^{\mathrm{t}}+\mathrm{MgO}\right)\right]$ vs. $\mathrm{SiO}_{2}$, and (d) MALI-index $\left(\mathrm{Na}_{2} \mathrm{O}+\mathrm{K}_{2} \mathrm{O}-\mathrm{CaO}\right)$ vs. $\mathrm{SiO}_{2}$ diagrams after Frost et al. (2001).

Major elements of GPG and PG form welldefined clusters and trends in Harker bivariate plots (figure 6), where $\mathrm{TiO}_{2}, \mathrm{P}_{2} \mathrm{O}_{5}, \mathrm{CaO}, \mathrm{Fe}_{2} \mathrm{O}_{3}{ }^{\mathrm{t}}$ and $\mathrm{Al}_{2} \mathrm{O}_{3}$ concentrations decrease monotonously with increasing amount of $\mathrm{SiO}_{2}$. The variation of $\mathrm{Na}_{2} \mathrm{O}$ however, is independent of the $\mathrm{SiO}_{2}$, and the $\mathrm{K}_{2} \mathrm{O}$ displays a weak positive correlation with $\mathrm{SiO}_{2}$. The overall pattern of decreasing $\mathrm{TiO}_{2}, \mathrm{Fe}_{2} \mathrm{O}_{3}{ }^{\mathrm{t}}$, $\mathrm{MgO}$ and $\mathrm{CaO}$ with increasing $\mathrm{SiO}_{2}$ is characteristic of fractionating granitic systems. The variation patterns of selected trace elements with respect to each other and $\mathrm{SiO}_{2}$ show simple fractionation trends (figure 7). $\mathrm{Ba}, \mathrm{Sr}$ and $\mathrm{Zr}$ generally decrease with increasing $\mathrm{SiO}_{2}$ for all samples of GPG and PG, whereas Rb shows little correlation with increasing silica. $\mathrm{Rb}$ and $\mathrm{Ba}$ are more enriched relative to $\mathrm{Sr}$, and overall $\mathrm{Rb} / \mathrm{Sr}$ ratios are high but comparatively higher in GPG (1.92-9.84) than PG $(2.02-3.87)$. The major and trace elements along with inter-elemental variation diagrams suggest a key role of fractional crystallisation processes during petrogenesis of these granites.

\subsection{Trace and Rare earth element patterns}

Chondrite normalised REE patterns reveal a distinction between GPG and PG (figure 8a and c), where samples of GPG show similar patterns with a lesser degree of REE fractionation $\left((\mathrm{La} / \mathrm{Yb})_{\mathrm{N}}=\right.$ 2.85-8.24) than $\mathrm{PG}$, which shows a higher degree of fractionation $\left((\mathrm{La} / \mathrm{Yb})_{\mathrm{N}}=6.88-58.12\right)$. The GPG has high $\Sigma$ REE (an average $153.85 \mathrm{ppm}$ ) content, and shows moderate LREE enrichment $\left((\mathrm{La} / \mathrm{Sm})_{\mathrm{N}}=1.85-3.56\right)$ with enriched but relatively flat $\operatorname{HREE}\left((\mathrm{Gd} / \mathrm{Yb})_{\mathrm{N}}=1.17-2.48\right)$ patterns and distinct negative Eu anomaly $\left(\mathrm{Eu} / \mathrm{Eu}^{*}=0.15-\right.$ 0.9) (figure 8a). PG, on the other hand, has relatively low $\Sigma$ REE (an average $148.40 \mathrm{ppm}$ ) contents and exhibits strongly fractionated REE patterns with enriched LREE $\left((\mathrm{La} / \mathrm{Sm})_{\mathrm{N}}=2.49\right.$ $6.08)$ and depleted HREE $\left((\mathrm{Gd} / \mathrm{Yb})_{\mathrm{N}}=2.21-9.43\right)$ patterns having concave upward shape, and show weak negative to moderate positive Eu anomaly $\left(\mathrm{Eu} / \mathrm{Eu}^{*}=0.48-1.05\right)$ (figure 8c). On the primitive mantle normalised multi-element spidergrams 

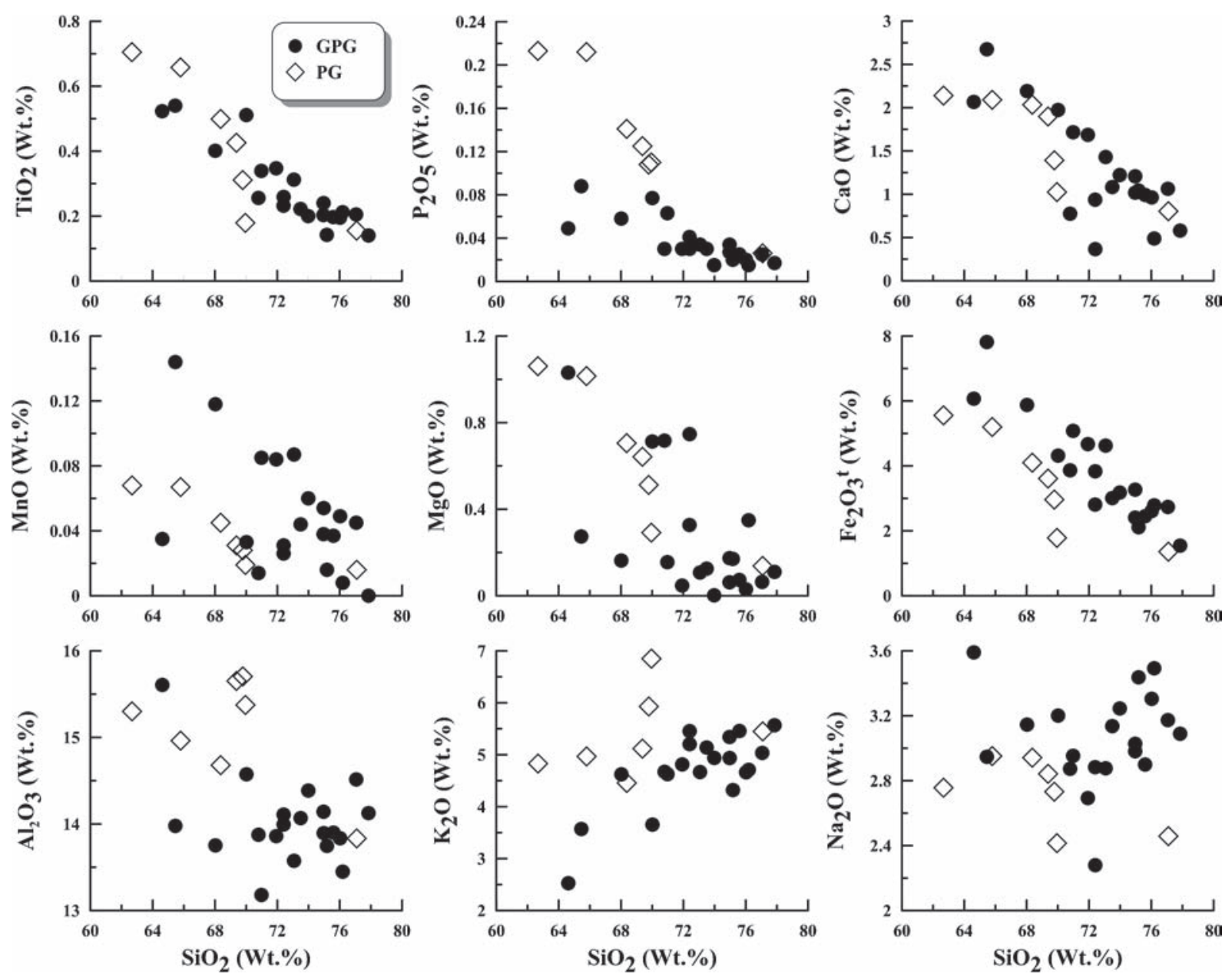

Figure 6. Harker variation diagrams of the major element oxides with $\mathrm{SiO}_{2}$ for the studied granites.

(figure $8 \mathrm{~b}$ and $\mathrm{d}$ ), samples of GPG and PG show similar patterns with more or less same degree of elemental enrichment. They show characteristic features common to A-type granites, viz., distinctive depletion of LILE's like Ba, Sr and HFSE's like $\mathrm{Nb}, \mathrm{P}$ and Ti relative to other trace elements.

\subsection{Classification}

All samples of GPG and PG plot in the field of A-type granites in the $\mathrm{Fe}^{*}$ vs. $\mathrm{SiO}_{2}$ diagram of Frost et al. (2001) (figure 5c). Also, Whalen et al. (1987) have proposed discrimination diagrams to distinguish A-type granites from the other variants like I-, S- and M-type granites. The variation of the major oxide ratios and trace elements like $\left(\mathrm{K}_{2} \mathrm{O}+\mathrm{Na}_{2} \mathrm{O}\right) / \mathrm{CaO}, \mathrm{FeO}^{\mathrm{t}} / \mathrm{MgO}, \mathrm{Zr}$ and $\mathrm{Nb}$ with $\mathrm{Ga} / \mathrm{Al}$ (figure $9 \mathrm{a}, \mathrm{b}, \mathrm{d}$ and e), show that all the samples clearly correspond to A-type granites. However, some less evolved samples lie at the boundary between the A-type and (I, S and M)type fields in the diagrams. In the $\left(\mathrm{K}_{2} \mathrm{O}+\mathrm{Na}_{2} \mathrm{O}\right) /$ $\mathrm{CaO}$ and $\mathrm{FeO}^{\mathrm{t}} / \mathrm{MgO}$ vs. $\mathrm{Zr}+\mathrm{Nb}+\mathrm{Ce}+\mathrm{Y}$ diagrams to discriminate fractionated granites from A-type granites (figure $9 \mathrm{c}$ and $\mathrm{f}$ ), the samples show a consistent A-type character. Moreover, some samples from both GPG and PG have a compositional overlap between A-type and fractionated granites $(\mathrm{FG})$. In $\mathrm{Rb}$ vs. $(\mathrm{Y}+\mathrm{Nb})$ tectonic discrimination diagram of Pearce et al. (1984) (figure 9g), the majority of GPG samples lie in the within-plate granite field whereas the PG samples straddle at the triple junction boundary of the within-plate, volcanic arc and syn-collisional granites. However, in $\mathrm{Y}$ vs. $\mathrm{Nb}$ (figure 9h) majority of samples are concentrated predominantly in the within-plate granite field while some samples of GPG fall in the field of volcanic arc plus syn-collisional granites. This also provides support for an A-type character as Atype is considered to be equivalent to within-plate granites (Whalen et al. 1987; Eby 1992).

\section{Discussion}

\subsection{Estimated temperature of magma}

Zircon saturation temperatures $\left(\mathrm{T}_{\mathrm{Zr}}\right)$ calculated from bulk rock compositions provide a simple and 

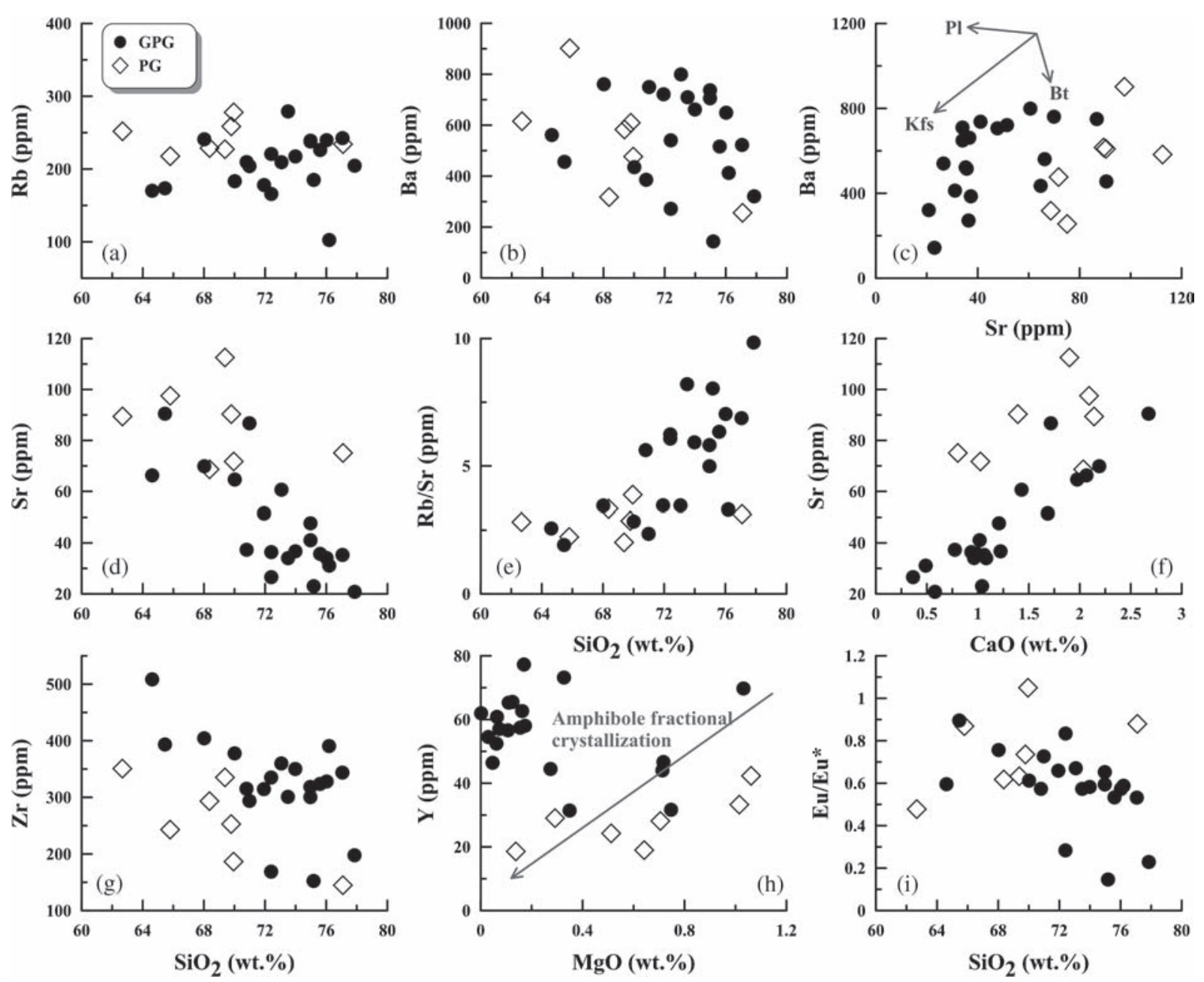

Figure 7. Binary diagrams of (a) $\mathrm{SiO}_{2}$ vs. $\mathrm{Rb},(\mathbf{b}) \mathrm{SiO}_{2}$ vs. $\mathrm{Ba}$, (c) $\mathrm{Sr}$ vs. $\mathrm{Ba}$, (d) $\mathrm{SiO}_{2}$ vs. $\mathrm{Sr},(\mathbf{e}) \mathrm{SiO} 2$ vs. $\mathrm{Rb} / \mathrm{Sr},(\mathbf{f}) \mathrm{CaO}$ vs. $\mathrm{Sr},(\mathrm{g}) \mathrm{SiO}_{2}$ vs. $\mathrm{Zr},(\mathbf{h}) \mathrm{MgO}$ vs. $\mathrm{Y}$ and (i) $\mathrm{SiO}_{2}$ vs. Eu/Eu* showing fractionation of feldspar, hornblende, zircon for the studied granites. The vectors in $\mathrm{Sr}$ vs. Ba plot are taken from Huang et al. (2012).

robust means of estimating magma temperatures (Watson and Harrison 1983; Miller et al. 2003). Watson and Harrison (1983) have established experimentally that the partition coefficient of zircon $\mathrm{D}^{\mathrm{Zr} \frac{\text { zircon }}{\mathrm{melt}}}$ is a function of the parameter $\mathrm{M}[(\mathrm{Na}+\mathrm{K}+2 \mathrm{Ca}) /(\mathrm{Al} * \mathrm{Si})$, all in cation fraction $]$ and temperature. Therefore, using this formula, the $\mathrm{Zr}$ thermometry yields the temperature at which the granites of study area formed, i.e., an average $\mathrm{T}_{\mathrm{Zr}}\left({ }^{\circ} \mathrm{C}\right) \sim 861$ for $\mathrm{GPG}$ and 835 for $\mathrm{PG}$ (table 1) (figure 9i). However, Miller et al. (2003) showed that the average $\mathrm{T}_{\mathrm{Zr}}$ for inheritance-rich granitoids are $766^{\circ} \mathrm{C}$ and inheritance-poor granitoids are $837^{\circ} \mathrm{C}$ because zirconium concentrations in inheritance-rich granitoids are uniformly lower than those of inheritance-poor intrusions. Consequently, calculated $\mathrm{T}_{\mathrm{Zr}}$ values are also lower for inheritance-rich granitoids. Therefore, the rocks in the study area are hot inheritance-poor granitoids, i.e., undersaturated in zircon at the source, with $\mathrm{T}_{\mathrm{Zr}}>800^{\circ} \mathrm{C}$ and the $\mathrm{T}_{\mathrm{Zr}}$ suggests a minimum initial magma temperature at the source.

\subsection{A-type granites: Reduced or oxidised nature?}

The granites in the study area have most of the petrological and geochemical characteristics associated with the definition of A-type granites (Loiselle and Wones 1979; Whalen et al. 1987; Eby 1990; Frost et al. 2001). They are amphibole-bearing and the bulk composition is characterised by low $\mathrm{CaO}$, Ba and $\mathrm{Sr}$, high $\mathrm{Na}_{2} \mathrm{O}+\mathrm{K}_{2} \mathrm{O}$ and $\mathrm{FeO}^{\mathrm{t}} /$ $\left(\mathrm{FeO}^{\mathrm{t}}+\mathrm{MgO}\right)$, high REE (except $\mathrm{Eu}$ ) and HFSE (Zr, Y and $\mathrm{Nb}$ ) values. They also have very high Zr saturation temperatures $\left(799-915^{\circ} \mathrm{C}\right)$ and lack of significant inherited zircon also support to an Atype character (Creaser et al. 1991). Overall, the granites are very similar in composition and mineralogy to the peraluminous A-type granites from Australia (Turner et al. 1992; King et al. 1997).

Dall'Agnol and de Oliveira (2007) have proposed a new classification scheme to separate calc-alkanine granites from A-type granites and oxidised A-type granites from reduced A-type granites. In the $\mathrm{CaO} /\left(\mathrm{FeO}^{\mathrm{t}}+\mathrm{MgO}+\mathrm{TiO}_{2}\right)$ vs. $\mathrm{CaO}+\mathrm{Al}_{2} \mathrm{O}_{3}$ 

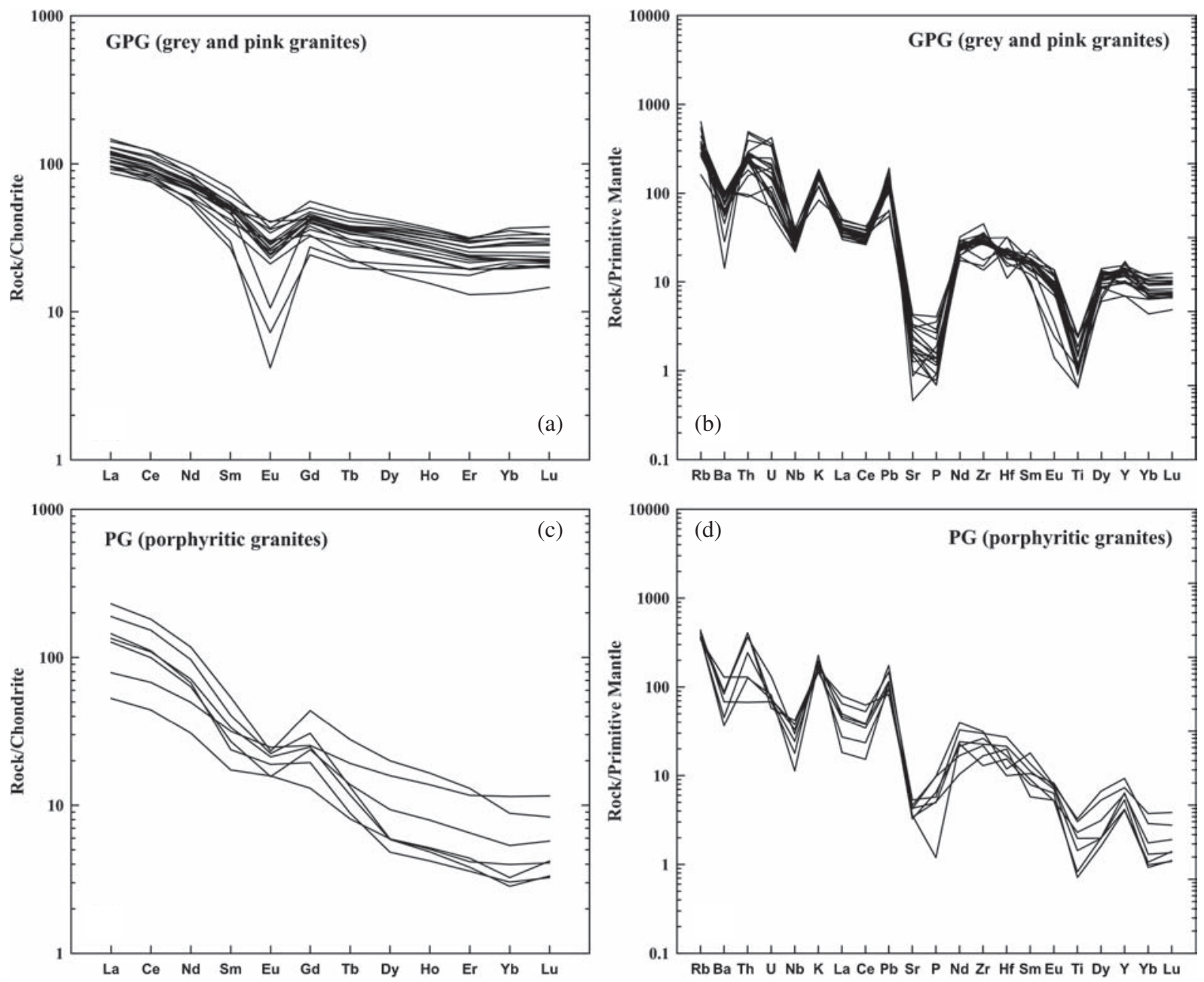

Figure 8. Chondrite normalised rare earth elements (REE) patterns (a and c) (normalisation value after McDonough and Sun 1995) and primitive mantle normalised trace element patterns (b and $\mathbf{d}$ ) (normalisation value after Sun and McDonough 1989).

and $\mathrm{CaO} /\left(\mathrm{FeO}^{\mathrm{t}}+\mathrm{MgO}+\mathrm{TiO}_{2}\right)$ vs. $\mathrm{Al}_{2} \mathrm{O}_{3}$ diagrams (figure $10 \mathrm{a}$ and $\mathrm{b}$ ), the granites fall mostly in the A-type granite field and they differ clearly from the calc-alkaline granites. In the $\mathrm{FeO}^{\mathrm{t}} /$ $\left(\mathrm{FeO}^{\mathrm{t}}+\mathrm{MgO}\right)$ vs. $\mathrm{Al}_{2} \mathrm{O}_{3}$ and $\mathrm{FeO}^{\mathrm{t}} /\left(\mathrm{FeO}^{\mathrm{t}}+\mathrm{MgO}\right)$ vs. $\mathrm{Al}_{2} \mathrm{O}_{3} /\left(\mathrm{K}_{2} \mathrm{O} / \mathrm{Na}_{2} \mathrm{O}\right)$ diagrams (figure $10 \mathrm{c}-\mathrm{d}$ ), the majority of GPG samples fall in the field of reduced A-type granite, though some samples also fall in the oxidised A-type granite field. The samples of $\mathrm{PG}$, on the contrary, fall in the field of oxidised A-type granites.

\subsection{Petrogenesis}

\subsubsection{Implications from Trace and REEs}

The Chondrite normalised REE patterns of GPG show less fractionated REE patterns, with enriched LREE and HREE and strong negative Eu anomaly (figure 8a). These features can be explained by constraining the source rock to a basic plagioclase rich-garnet free composition. Melting of such source rock will result in melts with negative Eu anomalies and enriched HREE with low LREE/HREE ratios (Barker 1979; Martin 1999). These features can further be explained by the fractional crystallisation of plagioclase from the melt followed by late stage hornblende crystallisation to deplete the LREE and impart the low LREE/HREE ratios (Arth and Barker 1976; Barker 1979). PG, on the other hand, shows highly fractionated REE patterns (high LREE/HREE ratios) with weak negative to moderate positive $\mathrm{Eu}$ anomaly (figure 8c). The incompatible behaviour of Eu indicates melting of a source with little plagioclase, whereas the depletion of HREE is predominantly caused by low degrees of melting of garnet-rich sources which retain and depletes the melt in HREE (Drummond and Defant 1990; Martin et al. 2005). The other probable explanation for the depleted HREE in PG could be the late stage fractionation of heavy mineral phases like zircon, allanite, sphene, apatite and monazite because of their extremely high partition coefficients for HREE (Peter Gromet and 

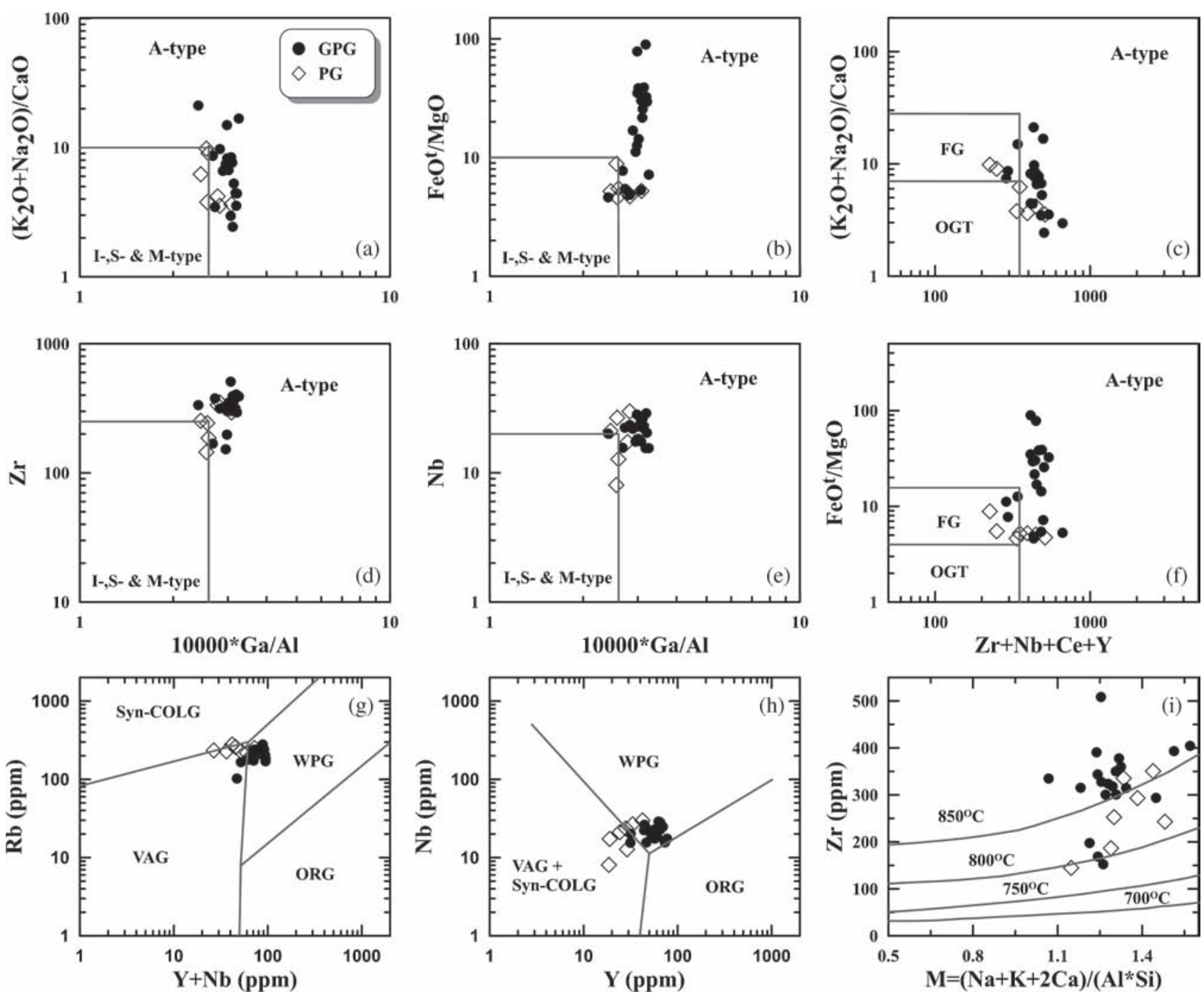

Figure 9. (a-f) Granitoid discrimination diagrams from Whalen et al. (1987) for GPG and PG samples. FG-field for fractionated I-type granitoids; OGT-field for I-, S- and M-type granitoids, (g-h) Y+Nb vs. Rb and Y vs. Nb tectonic discrimination diagrams of Pearce et al. (1984). Abbreviations of fields; VAG: volcanic arc granites; WPG: within plate granites; Syn-COLG: Syn-collisional granites; ORG: ocean ridge granites; Post-COLG: post-collision granites, (i) Zr concentrations in granites plotted against the cationic ratio $\mathrm{M}$. The lines marked $700^{\circ}-850^{\circ} \mathrm{C}$ indicate the proportions of $\mathrm{Zr}$ that can be dissolved in granitic melts of different compositions, expressed by the M parameter (Miller et al. 2003; Watson and Harrison 1983).

Silver 1983; Rollinson 1993). The $\mathrm{Eu} / \mathrm{Eu}^{*}$ vs. $\mathrm{SiO}_{2}$ plot (figure 7i) shows that the size of negative Eu anomalies decreases with increasing differentiation for GPG and vice-versa for PG. The reduction in size of the negative Eu anomalies for PG and concave HREE pattern, could possibly be attributed to hornblende fractionation because $\mathrm{Eu}$ has a lower partition coefficient than most MREE and HREE for hornblende in felsic rocks (Green 1994). High concentrations of $\mathrm{Th}, \mathrm{U}$ and $\mathrm{Pb}$ in GPG and PG are indicative of the involvement of crustal components in their genesis (figure $8 \mathrm{~b}$ and $\mathrm{d}$ ). Negative anomalies of $\mathrm{Nb}, \mathrm{P}$ and $\mathrm{Ti}$ are considered to be a characteristic feature of a subduction-related environment. The high values of Y for GPG, also suggest their formation by melting of a garnet free source. Thus, the low Sr, high Y and HFSE contents with negative Eu anomaly indicate an origin at a shallower depth for GPG rocks (low $\mathrm{P}-\mathrm{T}$ conditions) when compared to $\mathrm{PG}$ rocks which originate relatively at deeper levels.

\subsubsection{Fractional crystallisation}

The linear geochemical trends of major and trace elements illustrated by decreasing $\mathrm{Al}_{2} \mathrm{O}_{3}, \mathrm{CaO}$, $\mathrm{MgO}, \mathrm{Fe}_{2} \mathrm{O}_{3}{ }^{\mathrm{t}}, \mathrm{TiO}_{2}, \mathrm{P}_{2} \mathrm{O}_{5}, \mathrm{Ba}, \mathrm{Sr}$ and $\mathrm{Zr}$ and increasing $\mathrm{Rb}$ and $\mathrm{Rb} / \mathrm{Sr}$ with increasing silica coupled with the similarity and parallel nature of the normalised REE patterns and depletion in $\mathrm{Ba}, \mathrm{Sr}$, $\mathrm{Nb}, \mathrm{P}, \mathrm{Ti}$ and $\mathrm{Eu}$ in the spidergrams, suggest a 

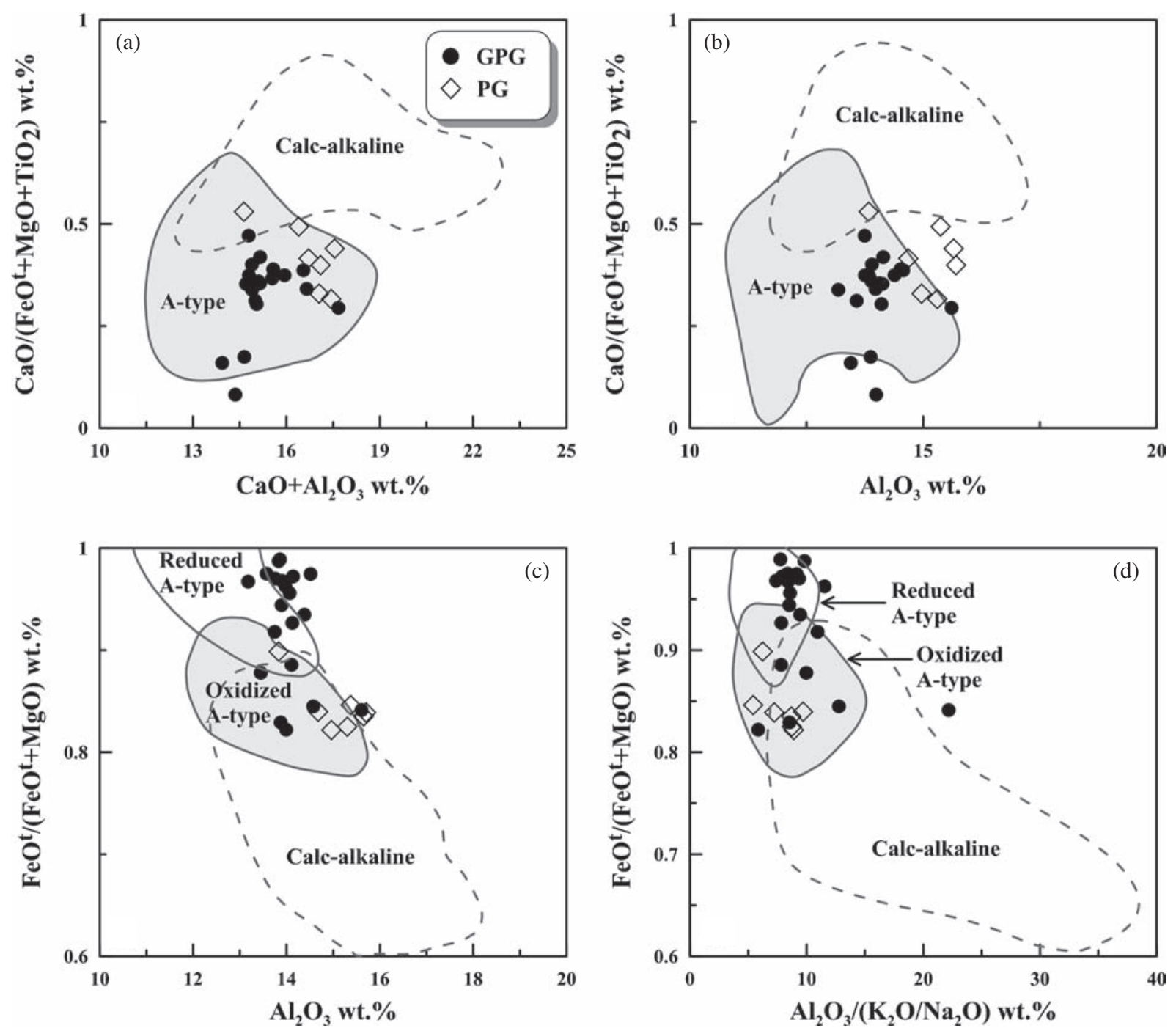

Figure 10. Whole-rock (a) $\mathrm{CaO} /\left(\mathrm{FeO}^{\mathrm{t}}+\mathrm{MgO}+\mathrm{TiO}_{2}\right)$ vs. $\mathrm{CaO}+\mathrm{Al}_{2} \mathrm{O}_{3},(\mathbf{b}) \mathrm{CaO} /\left(\mathrm{FeO}^{\mathrm{t}}+\mathrm{MgO}+\mathrm{TiO}_{2}\right)$ vs. $\mathrm{Al}_{2} \mathrm{O}_{3},(\mathbf{c}) \mathrm{FeO}^{\mathrm{t}} /$ $\left(\mathrm{FeO}^{\mathrm{t}}+\mathrm{MgO}\right)$ vs. $\mathrm{Al}_{2} \mathrm{O}_{3}$, and $(\mathbf{d}) \mathrm{FeO}^{\mathrm{t}} /\left(\mathrm{FeO}^{\mathrm{t}}+\mathrm{MgO}\right)$ vs. $\mathrm{Al}_{2} \mathrm{O}_{3} /\left(\mathrm{K}_{2} \mathrm{O} / \mathrm{Na}_{2} \mathrm{O}\right)$ discrimination diagrams with compositional fields from Dall'Agnol and de Oliveira (2007).

major role of crystal fractionation during the evolution of these rocks. The decrease in $\mathrm{P}_{2} \mathrm{O}_{5}$, $\mathrm{Fe}_{2} \mathrm{O}_{3}{ }^{\mathrm{t}}$ and $\mathrm{TiO}_{2}$ with increasing $\mathrm{SiO}_{2}$ (figure 6), along with strong negative $\mathrm{Nb}, \mathrm{P}$ and $\mathrm{Ti}$ anomalies (figure $8 \mathrm{~b}$ and $\mathrm{d}$ ) suggests fractionation of apatite and $\mathrm{Fe}-\mathrm{Ti}$ oxides for all samples. The depletion of $\mathrm{U}$ relative to $\mathrm{Th}$ is observed in the spidergrams of GPG and PG, indicating the fractionation of either zircon or allanite. The presence of negative $\mathrm{Eu}, \mathrm{Sr}$ and $\mathrm{Ba}$ anomalies in these rocks are indicative of fractionation of both plagioclase and K-feldspar. The pronounced negative Eu anomaly in the REE patterns of GPG require extensive fractionation of plagioclase when compared to $\mathrm{PG}$ and is also supported by negative correlation of $\mathrm{Al}_{2} \mathrm{O}_{3}$ and $\mathrm{CaO}$ with $\mathrm{SiO}_{2}$ (figure 6), and the positive one between $\mathrm{SiO}_{2}$ vs. $\mathrm{Rb} / \mathrm{Sr}$ and $\mathrm{CaO}$ vs. $\mathrm{Sr}$ (figure $7 \mathrm{e}$ and $\mathrm{f}$ ). However, the increase of $\mathrm{Ba}$ with $\mathrm{Sr}$ (figure 7c) suggests a systematic removal of K-feldspar, biotite and plagioclase from the melt for all samples. In addition, the fractionation of mafic phases particularly hornblende is suggested by the negative correlation of $\mathrm{MgO}$ and $\mathrm{Fe}_{2} \mathrm{O}_{3}{ }^{\mathrm{t}}$ with $\mathrm{SiO}_{2}$ (figure 6) and the positive correlation between $\mathrm{MgO}$ vs. $\mathrm{Y}$ (figure $7 \mathrm{~h}$ ) and $\mathrm{A} / \mathrm{CNK}$ vs. $\mathrm{SiO}_{2}$ (figure not shown) which is predominant in PG. The decreasing $\mathrm{Zr}$ content with increasing differentiation (figure $7 \mathrm{~g}$ ) for all samples in accordance with their zircon saturation temperatures indicates that with progressive fractionation, the solubility of $\mathrm{Zr}$ decreases in the melt because of falling temperature and, consequently, zircon is being removed from the melt (King et al. 1997).

\subsubsection{Magma source}

A number of petrogenetic schemes have been proposed for the origin of A-type granites involving 
the crustal and mantle sources that reflect their distinct and diversified compositions (Kemp and Hawkesworth 2003). However, Loiselle and Wones (1979) originally suggested that A-type granites are formed by fractionation of mantle-derived alkali basalt with or without crustal interaction, whereas Eby (1990) showed that A-type granitic melts can also be formed from mixing between crustal and mantle sources. Therefore, three main modes of origin have been proposed by Frost and Frost (2011) on the basis of earlier studies to produce ferroan granitic compositions: (1) partial melting of quartz-feldspathic crustal rocks, (2) differentiation of tholeiitic or alkali basaltic magma, (3) a combination of the first two models, in which differentiating basaltic magmas assimilate crustal rocks.

From the above discussions, it is clear that GPG are ferroan, calc-alkalic, metaluminous to peraluminous reduced A-type granites though some samples show oxidised nature too. These features are consistent with their derivation from the low-pressure partial melting of quartz-feldspathic igneous sources with a metasedimentary rock contribution (Dall'Agnol and de Oliveira 2007), or alternatively from differentiation of tholeiitic sources with significant crustal contamination (Frost and Frost 2011). In contrast to GPG, less evolved PG granites are ferroan, alkali-calcic to calc-alkalic peraluminous showing oxidised A-type characteristics, which can be attributed to partial melting of lower crustal quartz-feldspathic igneous sources under relatively oxidising conditions with appreciable water contents ( $\geq 4$ wt.\%) (Dall'Agnol and de Oliveira 2007), or can be interpreted to form by partial melting of felsic granulite (Frost and Frost 2011). Eby $(1990,1992)$ has shown that the $\mathrm{Y} / \mathrm{Nb}$ ratios are relatively constant and have very little or negligible effect during fractionation of Atype magmas. Therefore, this ratio can be regarded as a key to identify the sources of magma. Magmas with $\mathrm{Y} / \mathrm{Nb}<1.2$ are derived from the mantle sources, whereas higher $\mathrm{Y} / \mathrm{Nb}(>1.2)$ ratios imply solely crustal sources or mixed sources. The average $\mathrm{Y} / \mathrm{Nb}$ ratios of GPG (2.70) and PG (1.52) are greater than 1.2 , implying their derivation either from a crustal or mixed source.

The high-temperature nature of GPG and PG $\left(\mathrm{T}_{\mathrm{zr}}: \mathrm{GPG}_{\mathrm{avg}} \sim 861^{\circ} \mathrm{C}\right.$ and $\left.\mathrm{PG}_{\mathrm{avg}} \sim 835^{\circ} \mathrm{C}\right)$ also suggest that they have been generated either in the lower crustal or upper mantle region. The heat required to melt the parent rocks at such high temperatures must be provided by the mantle-derived melts either directly or indirectly, i.e., they may only provide the necessary heat for crustal melting or they may contribute mass through differentiation and assimilation of crustal melts (Frost and Frost 2011). Also, the presence of the differentiated basalt-andesite-rhyolite suite reported from the northern and eastern vicinity of Gaya (Ahmad and Wanjari 2009; Saikia et al. 2014; Wanjari et al. 2012) suggests an interaction of hot mafic magma with the crust.

To put further constraints on the three potential sources for A-type magmatism, we use the relationship of $\mathrm{Y} / \mathrm{Nb}$ with $\mathrm{Th} / \mathrm{Nb}$, Th/Ta and $\mathrm{Ce} / \mathrm{Pb}$, as given by Moreno et al. (2014). These ratios provide an efficient discrimination between the continental crust, oceanic island and convergent margin rocks. On $(\mathrm{Y} / \mathrm{Nb})_{\mathrm{N}}$ vs. $(\mathrm{Th} / \mathrm{Nb})_{\mathrm{N}}$ diagram (figure 11a), all samples lie within the field of convergent margin magmatism plotting close to, or inside, the field of continental crust estimates. These relationships suggest an involvement of a continental crust component in the genesis of both GPG and PG which are formed in the convergent
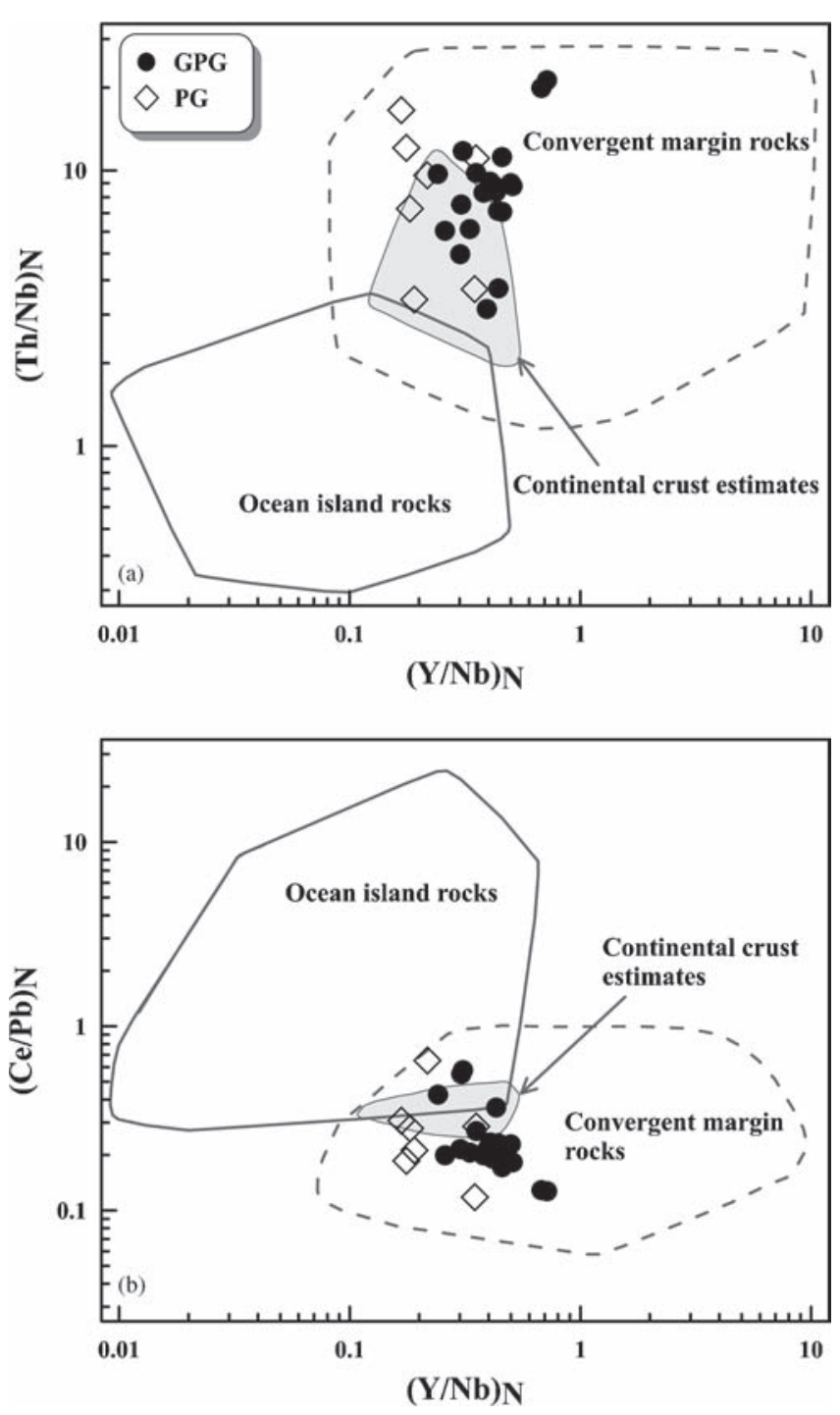

Figure 11. (a) $(\mathrm{Y} / \mathrm{Nb})_{\mathrm{N}}$ vs. $(\mathrm{Th} / \mathrm{Nb})_{\mathrm{N}}$ and $(\mathbf{b})(\mathrm{Y} / \mathrm{Nb})_{\mathrm{N}}$ vs. $(\mathrm{Ce} / \mathrm{Pb})_{\mathrm{N}}$ discrimination diagrams with compositional fields after Moreno et al. (2014). Values normalised to the silicate earth after McDonough and Sun (1995). 
margin, i.e., a collisional tectonic setting, also supported by negative $\mathrm{Nb}$ and positive $\mathrm{Pb}$ anomalies in spidergrams (figure $8 \mathrm{~b}$ and $\mathrm{d}$ ). Whereas on $(\mathrm{Y} / \mathrm{Nb})_{\mathrm{N}}$ vs. $(\mathrm{Ce} / \mathrm{Pb})_{\mathrm{N}}$ (figure $\left.11 \mathrm{~b}\right)$, all samples lie within the field of convergent margin magmatism but plot below the continental crust field. The significantly lower $(\mathrm{Ce} / \mathrm{Pb})_{\mathrm{N}}$ values presented by the granites suggest that this ratio was modified during magma differentiation. Thus, in the absence of isotopic constraints, the most likely origin for these granites seems to be the partial melting of crustal sources with variable mantle involvement, which is further evolved by the process of fractional crystallisation.

\subsubsection{Tectonic setting: Anorogenic or post-orogenic?}

A-type granites were originally defined as anorogenic granites by Loiselle and Wones (1979), but their exclusively anorogenic geotectonic settings
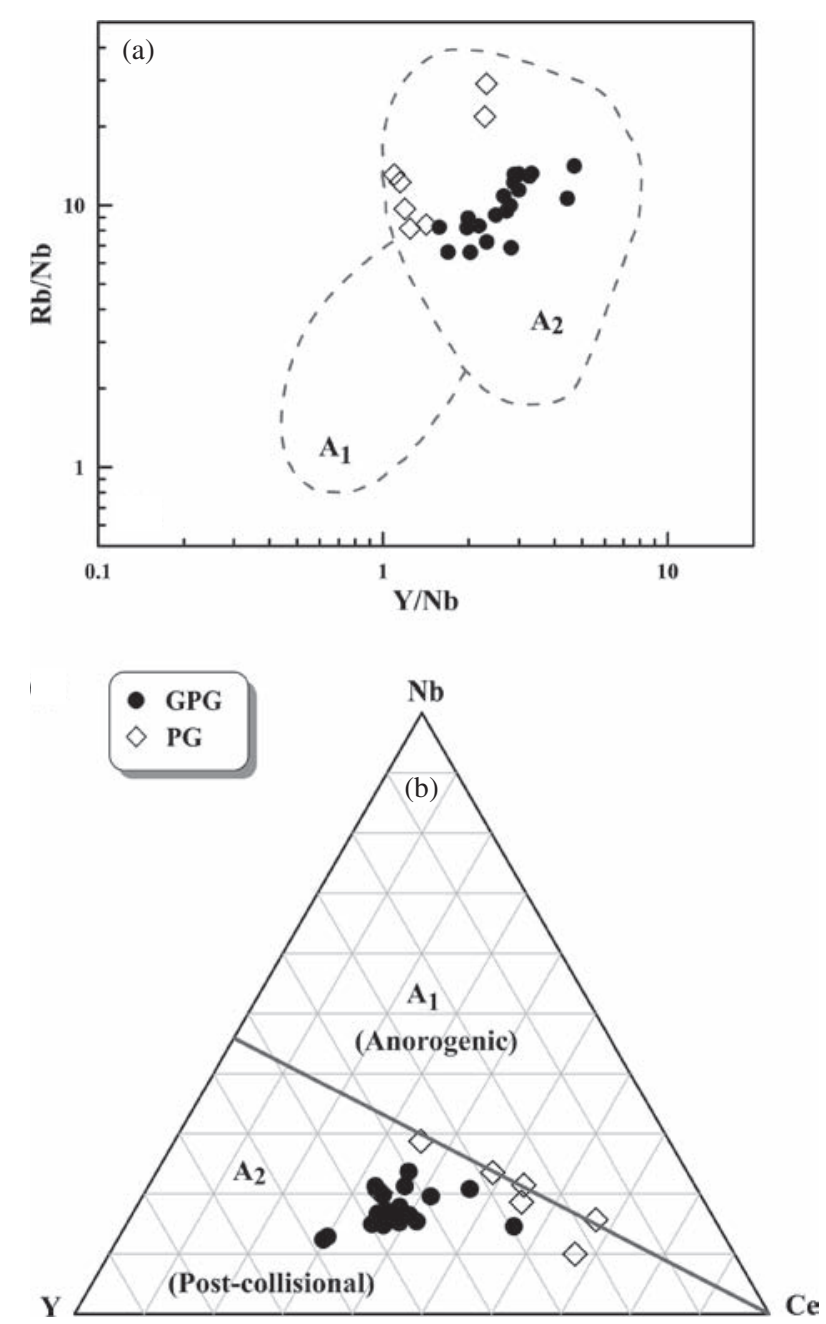

Figure 12. (a) $\mathrm{Y} / \mathrm{Nb}$ vs. $\mathrm{Rb} / \mathrm{Nb}$ and (b) $\mathrm{Y}-\mathrm{Nb}-\mathrm{Ce}$ subdiscriminant diagrams for A-type granites from Eby (1992). $\mathrm{A}_{1}$ : field for anorogenic A-type granites; $\mathrm{A}_{2}$ : field for postcollisional A-type granites. have been questioned and later studies suggested that they can be formed in both anorogenic and post-orogenic settings (Whalen et al. 1987; Eby 1992). Eby (1992) attempted to relate A-type granites with tectonic settings and further subdivided A-type into $\mathrm{A}_{1^{-}}$and $\mathrm{A}_{2}$-type granites. According to this classification based on $\mathrm{Y} / \mathrm{Nb}$ ratios, these two types represent different sources and tectonic settings, where $A_{1}(Y / N b<1.2)$ emplaced in an anorogenic setting, viz., continental rifts or intraplate environments and $\mathrm{A}_{2}(\mathrm{Y} / \mathrm{Nb}>1.2)$ in a post-orogenic setting, viz., continent-continent collision or island-arc setting. The $\mathrm{Y} / \mathrm{Nb}$ ratios of all the studied samples vary from 1.10-4.69. Therefore, the granites of the study area plot in the $\mathrm{A}_{2}$-type granite field on $\mathrm{Rb} / \mathrm{Nb}$ vs. $\mathrm{Y} / \mathrm{Nb}$ and $\mathrm{Y}-\mathrm{Ce}-\mathrm{Nb}$ diagrams (figure $12 \mathrm{a}$ and $\mathrm{b}$ ) suggest a post-collisional tectonic setting.

\section{Conclusions}

The granites present in the study area comprises grey and pink granites (GPG) which are Hbl-Bt rich whereas porphyritic granites $(\mathrm{PG})$ are mainly Bt rich. Geochemically both the GPG and PG are ferroan, alkali-calcic to calc-alkalic, metaluminous to peraluminous, reduced to oxidised A-type granites. Coherent geochemical trends and similarities in the trace element behaviour along with certain differences in the REE pattern of GPG and PG suggest a key role of fractional crystallisation during petrogenesis of these granites. Trace element characteristics, REE and the tectonic discrimination diagrams reveal that they were derived from a predominant crustal source with a variable degree of mantle input. PG were produced from partial melting of quartz-feldspathic igneous sources under relatively oxidising conditions with appreciable water contents, emplaced at deeper levels than GPG. In comparison, GPG were emplaced at shallower levels and were produced from low-pressure partial melting of quartz-feldspathic igneous sources with a metasedimentary rock contribution with relatively low water activity. An $\mathrm{A}_{2}$-type affinity suggests that these granites are post-collisional but not anorogenic. Further constraining of the magma sources and the spatiotemporal relationship of the granites can only be revealed by studying isotopic ratios.

\section{Acknowledgements}

The authors thank the Head of the Department of Geology, University of Delhi for all the facilities provided during this study, especially XRF analysis. We thank Prof. A K Choudhary from IIT Roorkee for ICP-MS analysis. They also thank 
the editor, Prof. Pulak Sengupta and the anonymous reviewers for their constructive reviews and useful suggestions, which helped in improving the manuscript. We thank all our lab colleagues for their cooperation and support.

\section{References}

Ahmad M and Wanjari N 2009 Volcano-sedimentary sequence in the Munger-Rajgir metasedimentary belt, Gaya district, Bihar; Indian J. Geosci. 63 351-360.

Arth J G and Barker F 1976 Rare-earth partitioning between hornblende and dacitic liquid and implications for the genesis of trondhjemitic-tonalitic magmas; Geol. 4 $534-536$.

Balaram V, Ramesh S L and Anjaiah K V 1996 New trace element and REE data in thirteen GSF reference samples by ICP-MS; Geostandard. Newslett. 20 71-78.

Banerji A 1991 Presidential address, Geology of the Chhotanagpur region; Indian J. Geol. 63 275-282.

Barker F 1979 Trondhjemite: Definition, environment and hypotheses of origin; Trondhjemites, Dacites and Related Rocks 6 1-12.

Bhattacharya B 1976 Metamorphism of the Precambrian rocks of the central part of Santhal Parganas district, Bihar; Quart. J. Geol. Min. Met. Soc. India 48 183-196.

Bhattacharya S, Chaudhary A K and Basei M 2012 Original nature and source of khondalites in the Eastern Ghats Province, India; Geol. Soc. London, Spec. Publ. 365 147-159.

Chatterjee N, Crowley J L and Ghose N C 2008 Geochronology of the $1.55 \mathrm{Ga}$ Bengal anorthosite and Grenvillian metamorphism in the Chotanagpur gneissic complex, eastern India; Precamb. Res. 161 303-316.

Chatterjee N and Ghose N C 2011 Extensive Early Neoproterozoic high-grade metamorphism in North Chotanagpur Gneissic Complex of the Central Indian Tectonic Zone; Gondwana Res. 20 362-379.

Creaser R A, Price R C and Wormald R J 1991 A-type granites revisited: Assessment of a residual-source model; Geol. 19 163-166.

Dall'Agnol R and de Oliveira D C 2007 Oxidized, magnetiteseries, rapakivi-type granites of Carajás, Brazil: Implications for classification and petrogenesis of A-type granites; Lithos 93 215-233.

Das B 1967 On the lithological sequence and overall structure of the rocks around Rajgir, Bihar; Bull. Geol. Soc. India 4 46-49.

Drummond M S and Defant M Z 1990 A model for Trondhjemite-Tonalite-Dacite genesis and crustal growth via slab melting: Archean to modern comparisons; J. Geophys. Res.: Solid Earth 95 21503-21521.

Dunn J A 1929 The geology of north Singhbhum including parts of Ranchi and Manbhum districts; Geological Survey of India Memoir.

Eby G N 1990 The A-type granitoids: A review of their occurrence and chemical characteristics and speculations on their petrogenesis; Lithos 26 115-134.

Eby G N 1992 Chemical subdivision of the A-type granitoids: Petrogenetic and tectonic implications; Geol. 20 641-644.

Frost B R, Barnes C G, Collins W J, Arculus R J, Ellis D J and Frost C D 2001 A geochemical classification for granitic rocks; J. Petrol. 42 2033-2048.

Frost C D and Frost B R 2011 On ferroan (A-type) granitoids: Their compositional variability and modes of origin; J. Petrol. 52 39-53.
Frost C D and Frost B R 2013 Proterozoic ferroan feldspathic magmatism; Precamb. Res. 228 151-163.

Ghose N 1983 Geology, tectonics and evolution of the Chhotanagpur granite gneiss complex, eastern India; Structure and Tectonics of Precambrian Rocks of India; Recent Res. Geol. 10 211-247.

Ghose N 1992 Chhotanagpur gneiss-granulite complex, eastern India: Present status and future prospect; Indian J. Geol. 64 100-121.

Ghose N C and Mukherjee D 2000 Chotanagpur gneissgranulite complex, eastern India - a kaleidoscope of global events; Platinum Jubilee Commemoration Volume, Indian School of Mines, Dhanbad Institute of Geoexploration and Environment, Monograph 2, Patna.

Green T H 1994 Trace-element partitioning with application to magmatic processes experimental studies of traceelement partitioning applicable to igneous petrogenesis Sedona 16 years later; Chem. Geol. 117 1-36.

GSI 2000 Eastern Nepal Himalaya and Indo-Gangetic Plains of Bihar; In: Seismotectonics Atlas of India and its Environs (eds) Narula P L, Acharyya S K and Banerjee J, Geological Survey of India, pp. 26-27.

Huang H, Zhang Z, Kusky T, Santosh M, Zhang S, Zhang D, Liu J and Zhao Z 2012 Continental vertical growth in the transitional zone between South Tianshan and Tarim, western Xinjiang, NW China: Insight from the Permian Halajun A1-type granitic magmatism; Lithos 155 49-66. Karmakar S, Bose S, Sarbadhikari A B and Das K 2011 Evolution of granulite enclaves and associated gneisses from Purulia, Chhotanagpur Granite Gneiss Complex, India: Evidence for 990-940 Ma tectonothermal event(s) at the eastern India cratonic fringe zone; J. Asian Earth Sci. 41 69-88.

Kemp A I S and Hawkesworth C J 2003 Granitic perspectives on the generation and secular evolution of the continental crust; In: Treatise on Geochemistry (ed.) Turekian H D H K, Pergamon, Oxford, pp. 349-410.

King P L, White A J R, Chappell B W and Allen C M 1997 Characterization and origin of aluminous A-type granites from the Lachlan Fold Belt, southeastern Australia; J. Petrol. 38 371-391.

Le Maitre R W, Streckeisen A, Zanettin B, Le Bas M, Bonin $\mathrm{B}$ and Bateman P 2002 Igneous rocks: A classification and glossary of terms; Recommendations of the International Union of Geological Sciences Subcommission on the Systematics of Igneous Rocks; Cambridge University Press.

Loiselle M and Wones D 1979 Characteristics and origin of anorogenic granites; Geol. Soc. Am., Abstracts with Programs, p. 468.

Mahadevan T 2002 Geology of Bihar and Jharkhand; GSI Publications 2.

Mallik A, Gupta S and Ray Barman T 1991 Dating of early Precambrian granite-greenstone complex of the eastern Indian Precambrian shield with special reference to the Chotanagpur granite gneiss complex; Rec. Geol. Surv. India 125 20-21.

Maniar P D and Piccoli P 1989 Tectonic discrimination of granitoids; Geol. Soc. Am. Bull. 101 635-643.

Martin H 1999 Adakitic magmas: Modern analogues of Archaean granitoids; Lithos 46 411-429.

Martin H, Smithies R H, Rapp R, Moyen J F and Champion D 2005 An overview of adakite, tonalite-trondhjemitegranodiorite (TTG), and sanukitoid: Relationships and some implications for crustal evolution; Lithos $\mathbf{7 9}$ $1-24$.

Mazumdar S K 1988 Crustal evolution of the Chhotanagpur gneissic complex and the mica belt of Bihar; Precambrian of the Eastern Indian Shield; Geol. Soc. India Memoir $\mathbf{8}$ 49-84. 
McDonough W F and Sun S S 1995 The composition of the Earth; Chem. Geol. 120 223-253.

Miller C F, McDowell S M and Mapes R W 2003 Hot and cold granites? Implications of zircon saturation temperatures and preservation of inheritance; Geol. 31 $529-532$.

Moreno J A, Molina J F, Montero P, Abu Anbar M, Scarrow J H, Cambeses A and Bea F 2014 Unraveling sources of A-type magmas in juvenile continental crust: Constraints from compositionally diverse Ediacaran post-collisional granitoids in the Katerina Ring Complex, southern Sinai, Egypt; Lithos 192-195 56-85.

Nesbitt H W and Young G M 1982 Early Proterozoic climates and plate motions inferred from major element chemistry of lutites; Nature 299 715-717.

O'connor J 1965 A classification for quartz-rich igneous rocks based on feldspar ratios; US Geol. Surv. Prof. Paper $B 525$ 79-84.

Pandey B, Gupta J and Lall Y 1986 Whole rock and mineral $\mathrm{Rb}-\mathrm{Sr}$ isochron ages for the granites from bihar Mica Belt of Hazaribagh, Bihar, India; Indian J. Earth Sci. 13 157-162.

Pearce J A, Harris N B W and Tindle A G 1984 Trace element discrimination diagrams for the tectonic interpretation of granitic rocks; J. Petrol. 25 956-983.

Peter Gromet L and Silver L T 1983 Rare earth element distributions among minerals in a granodiorite and their petrogenetic implications; Geochim. Cosmochim. Acta 47 925-939.

Rao M R 1973 The subsurface geology of the Indo-Gangetic plains; Geol. Soc. India 14 217-242.

Ray Barman T and Bishui P 1994 Dating of Chotanagpur gneissic complex of eastern Indian Precambrian shield; Record Geol. Surv. India 127 25-27.

Rollinson H R 1993 Using geochemical data: Evaluation, presentation, interpretation; Longman, London.

Saikia A, Gogoi B, Ahmad M and Ahmad T 2014 Geochemical constraints on the evolution of mafic and felsic rocks in the Bathani volcanic and volcano-sedimentary sequence of Chotanagpur Granite Gneiss Complex; J. Earth Syst. Sci. 123 959-987.
Sanyal S and Sengupta P 2012 Metamorphic evolution of the Chotanagpur Granite Gneiss Complex of the East Indian Shield: Current status; Geol. Soc. London, Spec. Publ. 365 117-145.

Sarkar A 1988 Tectonic evolution of the Chhotanagpur plateau and the Gondwana basins in Eastern India: An interpretation based on supra-subduction geological processes; Precambrian of the Eastern Indian Shield; Mem. Geol. Soc. India, pp. 127-146.

Sastri V, Bhandari L, Raju A and Datta A 1971 Tectonic framework and subsurface stratigraphy of the Ganga basin; Geol. Soc. India 12 222-233.

Sharma R 2009 Cratons and fold belts of India; Springer.

Siivola J and Schmid R 2007 List of mineral abbreviations - Recommendations by the IUGS Subcommission on the Systematics of Metamorphic Rocks; http://www.bgs.ac. uk/scmr/home.html.

Sun S S and McDonough W F 1989 Chemical and isotopic systematics of oceanic basalts: Implications for mantle composition and processes; Geol. Soc. London, Spec. Publ. 42 313-345.

Turner S P, Foden J D and Morrison R S 1992 Derivation of some A-type magmas by fractionation of basaltic magma: An example from the Padthaway Ridge, South Australia; Lithos 28 151-179.

Valdiya K S 1976 Himalayan transverse faults and folds and their parallelism with subsurface structures of North Indian plains; Tectonophys. 32 353-386.

Wanjari N R, Chaturvedi R and Mahanta D N 2012 Specialised thematic mapping in Munger-Rajgir Group of rocks to examine structural and stratigraphic set up in and around Gaya-Rajgir areas in parts of Gaya, Nawada and Jahanabad districts of Bihar; Unpublished report, GSI (F.S.: 2008-09, 2009-10).

Watson E B and Harrison T M 1983 Zircon saturation revisited: Temperature and composition effects in a variety of crustal magma types; Earth Planet. Sci. Lett. 64(2) 295-304.

Whalen J, Currie K and Chappell B 1987 A-type granites: geochemical characteristics, discrimination and petrogenesis; Contrib. Mineral. Petrol. 95 407-419. 